Rochester Institute of Technology

RIT Scholar Works

Theses

8-2014

Heterogeneous Photonic Network-on-Chip with Dynamic Bandwidth Allocation

Ankit Himanshu Shah

Follow this and additional works at: https://scholarworks.rit.edu/theses

Recommended Citation

Shah, Ankit Himanshu, "Heterogeneous Photonic Network-on-Chip with Dynamic Bandwidth Allocation" (2014). Thesis. Rochester Institute of Technology. Accessed from

This Thesis is brought to you for free and open access by RIT Scholar Works. It has been accepted for inclusion in Theses by an authorized administrator of RIT Scholar Works. For more information, please contact ritscholarworks@rit.edu. 


\title{
Heterogeneous Photonic Network-on-Chip with Dynamic Bandwidth Allocation \\ By
}

\section{Ankit Himanshu Shah}

A Thesis Submitted in Partial Fulfillment of the Requirements for the Degree of

\author{
Master of Science in Computer Engineering \\ Supervised by \\ Dr. Amlan Ganguly \\ Department of Computer Engineering \\ Kate Gleason College of Engineering \\ Rochester Institute of Technology \\ Rochester, NY \\ August, 2014
}

\section{Approved By:}

Dr. Amlan Ganguly

Primary Advisor - R.I.T. Dept. of Computer Engineering

Dr. Andres Kwasinski

Secondary Advisor - R.I.T. Dept. of Computer Engineering

Dr. Sonia Lopez Alarcon

Secondary Advisor - R.I.T. Dept. of Computer Engineering 


\section{Dedication}

Dedicated to my parents Mr. Himanshu Navinchandra Shah And Mrs. Mona Himanshu Shah 


\section{Acknowledgements}

I would like to express my great appreciation to my primary advisor Dr. Amlan Ganguly for his constant guidance, patience and support that he extended throughout the duration of this work. Dr Ganguly was always there to review my work and give valuable suggestions which always helped in keeping the work right on track.

Also, I would also like to thank Naseef Mansoor for all the valuable discussions and my parents for their encouragement and heartfelt support during the course of this work. 


\section{Abstract}

Advancements in the field of chip fabrication has facilitated in integrating more number of transistors in a given area which has lead to an era of multi-core processors. Future multi-core chips or chip multiprocessors (CMPs) will have hundreds of heterogeneous components including processing engines, custom logic, GPU units, programmable fabrics and distributed memory. Such multi-core chips are expected to run varied multiple parallel workloads simultaneously. Hence, different communicating cores will require different bandwidths leading to the necessity of a heterogeneous Network-on-Chip (NoC) architecture. Simply over-provisioning for performance will invariably result in loss of power efficiency. On the other hand, recent research has shown that photonic interconnects are capable of achieving high-bandwidth and energy-efficient on-chip data transfer. In this paper we propose a dynamic heterogeneous photonic NoC ( $d$-HetPNOC) architecture with dynamic bandwidth allocation to achieve better performance and energy-efficiency compared to a homogeneous photonic NoC architecture with the same aggregate data bandwidth. 


\section{Contents}

Abstract

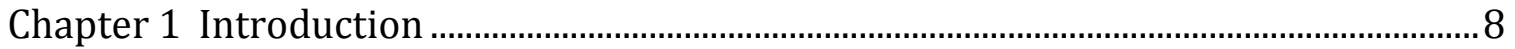

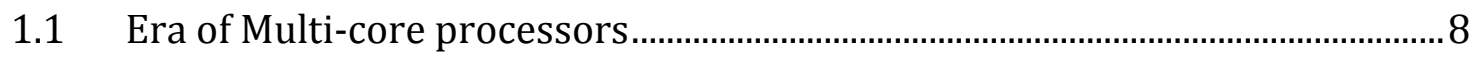

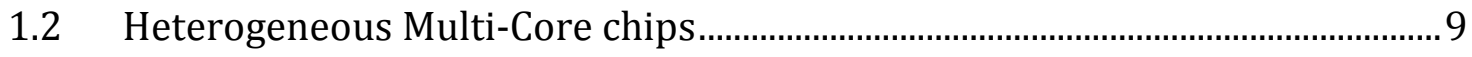

1.3 Interconnects in Multi-Core chips................................................................... 11

1.4 Network on Chip (NoC) paradigm .................................................................. 12

1.5 Emerging interconnects..................................................................................... 14

1.6 Thesis Contribution ........................................................................................... 17

Chapter 2 Related Work ............................................................................................... 19

$2.1 \quad$ Photonic Elements …………........................................................................... 19

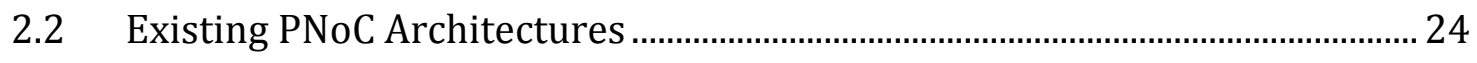

Chapter 3 Dynamic Heterogeneous Photonic NOC $(d$-HetPNoC) .................................. 27

$3.1 \quad$ Network Architecture ......................................................................................... 27

3.2 Dynamic Bandwidth Allocation (DBA) Mechanism ............................................ 29

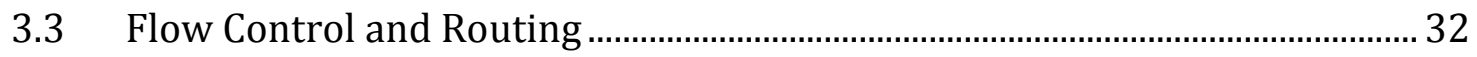

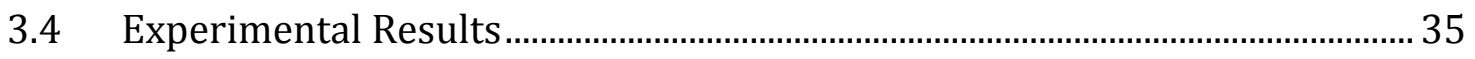

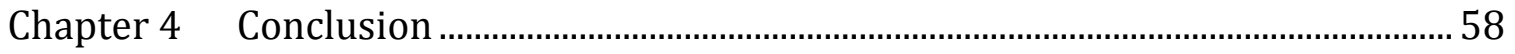

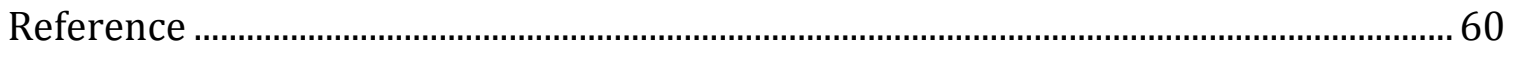




\section{List of figures}

Figure 1-1: Speedup of 1024B flit size over baseline (32B flit size) with benchmarks from CUDA SDK (upper case) [26] and Rodinia (lower case) [25] with number of kernel launches in parenthesis....................................................................................... 11

Figure 1-2: Network-on-Chip architecture ................................................................ 12

Figure 1-3: NoC switch architecture.......................................................................... 14

Figure 2-1: A basic photonic switch........................................................................... 22

Figure 2-2: Firefly Architecture: (a) Cross-bar between clusters of same assembly, (b) waveguide supporting inter-cluster crossbars. Reproduced form [20] ................ 25 Figure 2-3: Reservation-assisted Single Write Multiple Read. Adapted from [20].... 25 Figure 3-1: Dynamic bandwidth allocation enabled PNoC architecture........................ 28

Figure 3-2: Microarchitecture of photonic router......................................................... 34

Figure 3-3: Peak Bandwidth of Firefly PNoC and $d$-HetPNoC for uniform-random and skewed traffic patterns for (a) Bandwidth set 1 (Total Wavelengths $=64$ ) (b) Bandwidth set 2 (Total Wavelengths $=256$ ) (c) Bandwidth set 3 (Total Wavelengths $=512$ ).

Figure 3-4: Packet Energy of Firefly PNoC and $d$-HetPNoC for uniform-random and skewed traffic patterns for (a) BW Set 1 (Total Wavelengths $=64$ ) (b) BW set 2 (Total Wavelengths $=256$ ) (c) BW Set 3 (Total Wavelengths $=512$ ).

Figure 3-5: Peak Core Bandwidth and Packet Energy for Firefly PNoC for synthetic and real application based traffic scenarios. 45 Figure 3-6: Peak Core Bandwidth and Packet Energy for Firefly PNoC for synthetic and real application based traffic scenarios.

Figure 3-7: Comparison of (a) Peak Core Bandwidth (b) Energy Per Message of $d$ HetPNoC for BW Set 1 (Total Wavelengths $=64)$, BW Set 2 (Total Wavelengths $=$ 256) and BW Set 3 (Total Wavelengths = 512) for Uniform Random and Skewed Traffic Patterns

Figure 3-8: Effect of increase in total number of wavelengths on Peak Bandwidth and Area for $d$-HetPNoC for Skewed 3 traffic pattern. Figure 3-9: Effect of increase in total number of wavelengths on Energy per Message and Area for $d$-HetPNoC for Skewed 3 traffic pattern. 54 Figure 3-10: Comparison of (a) Peak Core Bandwidth (b) Energy Per Message of Firefly architecture for BW Set 1 (Total Wavelengths $=64$ ), BW Set 2 (Total Wavelengths $=256$ ) and BW Set 3 (Total Wavelengths $=512$ ) for Uniform Random and Skewed Traffic Patterns. 


\section{List of Tables}

Table 3-1: Frequency of communication for applications with different bandwidth for skewed traffic scenarios............................................................................................. 35 Table 3-2: Frequency of communication for applications with bandwidth set 1 for

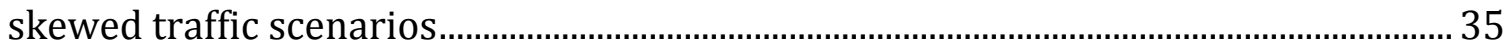

Table 3-3: Simulation Parameters................................................................................ 37

Table 3-4: Power or Energy dissipation of photonic components ................................. 37

Table 3-5: Energy of different photonic components...................................................... 41 


\section{Chapter 1 Introduction}

Modern sophisticated applications need more computational power and have been the driving force to build powerful computers. This was initially achieved by increasing the clock speed of the processor to get faster and computationally intensive computers. This however led to increased power consumption and increased dissipation of heat. In accordance with Moore's Law the number of transistors that are fabricated on a chip has doubled roughly every 18 months. With increase in the number of transistors and limit on the increase in clock speed, the cores on a chip were increased to exploit the parallelism in the computational requirement of an application. A significant amount of information is exchanged between these cores; hence interconnects between the cores plays an important role in multi-core chips.

\subsection{Era of Multi-core processors}

In the past the performance of the processors was improved by increasing the clock speeds. This helped speed up single-threaded, serial code. Increase in clock speed was achieved by increasing the depth of the pipeline. Deeper pipeline are no longer profitable as the flip-flops delay is comparable to the combinational logic delay. Another disadvantage of higher clock speeds was that Central Processing Units (CPUs) were hitting a power wall. Power dissipation in a processor is directly proportional to the clock speed at which the CPU operates i.e. CPU frequency. Higher clock speeds led to increased power dissipation. Moreover, the high frequency 
micro-architectures are not suited for some of the low power techniques that have been invented to reduce power.

The steady advancements in device and fabrication technology have enabled us to increase the transistor integration density [1]. Consequently the number of CPU cores that can be fabricated on the chip are increasing. Multi-core chips or chip multiprocessors (CMPs) provide parallel processing capabilities by running multiple threads at the same time consequently improving the processing time. Most of the modern day applications are multithreaded applications and they perform better on multi-core chip compared to uni-core chip. The multi-core chips can also be made to operate at lower frequency than the single core chip thus reducing the power dissipation in the chip. Moreover some of the cores in a multi-core chip can be turned off for tasks that require lesser computational power making them ideal for low power devices.

\subsection{Heterogeneous Multi-Core chips}

Decrease in feature size of transistor has resulted in increase in the integration density on a chip. Future CMPs will have hundreds to thousands of cores. To enable energy-efficient data-intensive computations, such cores will comprise of heterogeneous components like custom logic, CPUs, GPGPUs, reconfigurable hardware and distributed memory units [2]. Custom logic, GPGPUs and reconfigurable hardware would be used to speed up the parallelizable sections of the application while a conventional $\mathrm{CPU}$ will be used to execute the serial section of the application [3]. 
Such a heterogeneous system will require a heterogeneous interconnection fabric in which different channels have different bandwidths, to support non-uniform data traffic. Such heterogeneous interconnection fabric is envisioned to improve the communication between different heterogeneous components by reducing latency and energy required for communication. Traditional planar dielectric interconnects cannot deliver the dynamic bandwidth requirements of heterogeneous CMPs. Ability of interconnects to provide dynamic bandwidth will determine the system performance as the number of heterogeneous cores increases in a CMPs. Hence new interconnect options are studied to support the increasing number of heterogeneous cores in a multi-core Chip.

\subsubsection{Heterogeneous Bandwidth Requirement}

Heterogeneous multi-core system will require a heterogeneous interconnection fabric in which different channels have different bandwidths, to support nonuniform data traffic. It has been shown that traditional Networks-on-Chips (NoCs) with heterogeneous resource allocation can improve the performance-overhead trade-offs even with conventional metallic interconnects and mesh-based topologies [31]. Different classes of applications are shown to benefit from different types interconnects in [32]. Some applications benefit from low latency interconnects while others from high-bandwidth ones.

The heterogeneous bandwidth requirements in a future generation CMP can be understood from studying GPU-memory interactions, which will make up parts of its fabric. GPUs are highly bandwidth dependent [27], with drastic performance 
losses when the GPU-memory bandwidth is low. We have evaluated what is the maximum performance improvement that we can attain when we provide very high bandwidth. Figure 1-1 shows the speedup when the bandwidth of GPU-memory interconnects was increased by varying the flit size from 32B to $1024 \mathrm{~B}$ at $700 \mathrm{MHz}$. It is seen that despite the high bandwidth links most of the benchmarks show very modest performance improvement of less than below $1 \%$. On the other hand a few of the benchmarks show considerable speedup of up to $63 \%$. This indicates that differentiated interconnect channels are required in the same NoC fabric to harness their benefits for different types of workloads running in parallel on heterogeneous multi-core chips.

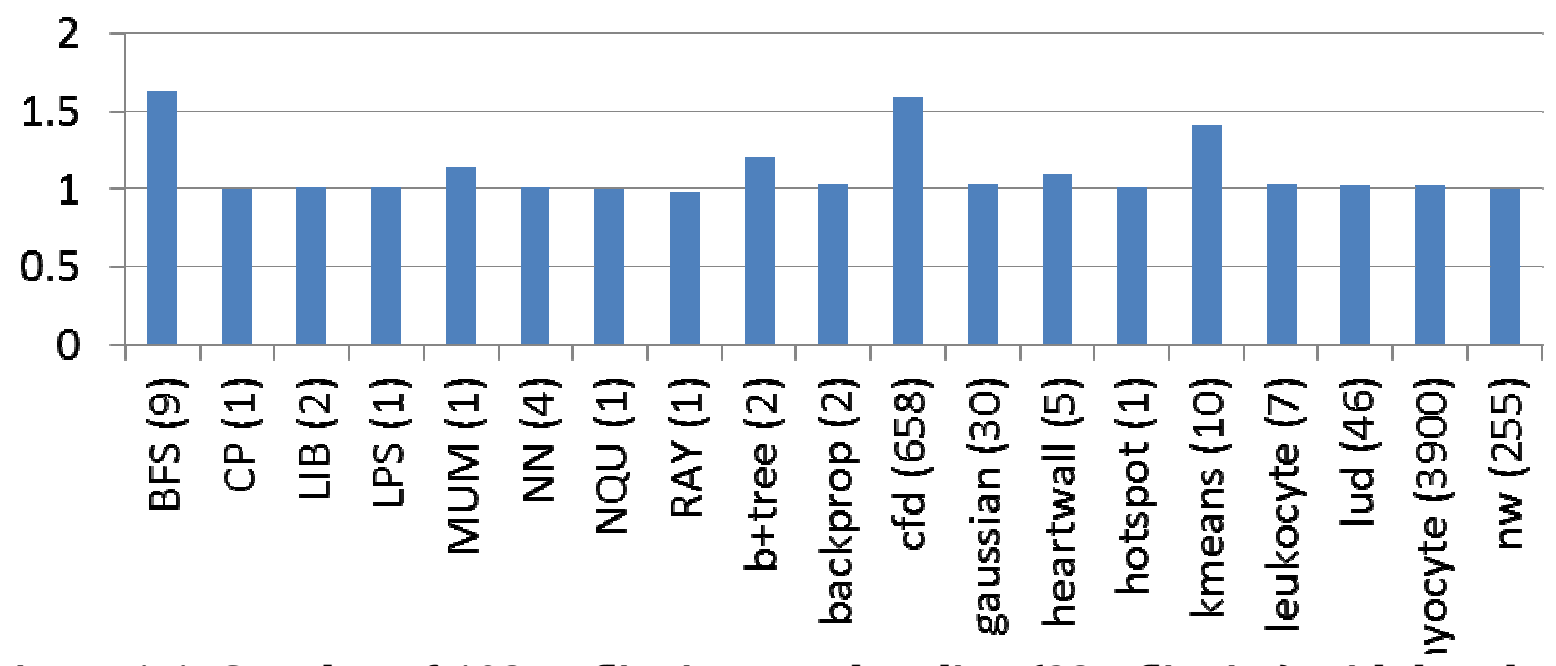

Figure 1-1: Speedup of 1024B flit size over baseline (32B flit size) with benchmarks from CUDA SDK (upper case) [26] and Rodinia (lower case) [25] with number of kernel launches in parenthesis

\subsection{Interconnects in Multi-Core chips}

Initially, CMPs used shared memory to communicate between the cores. However shared memory model is not scalable. With increase in the number of cores, sophisticated interconnects are needed for communication between the 
cores. Different interconnection architectures were developed for effective communication between the cores. Bus based, crossbar based, packet based interconnect architectures are some of the interconnection architectures. Intel and AMD uses bus based interconnection architecture. QuickPath interconnect from Intel is a point-to-point interconnect. QuickPath interconnect uses 20 bit wide bus running at $3.2 \mathrm{GHz}$ to provide uni-directional raw bandwidth of $12.8 \mathrm{~GB} / \mathrm{s}$ for communication between cores [4]. AMD's Hyper Transport 3.0 is a 32-bit wide bus running at $5.2 \mathrm{GHz}[5]$.

\subsection{Network on Chip (NoC) paradigm}

With increase in the number of cores on a chip, global on-chip communication will play an important role in overall performance of the chip. Using long electrical wires for global communication is unreliable because of increased crosstalk and noise sensitivity. Hence there is a need for an interconnection network for communication between the cores.

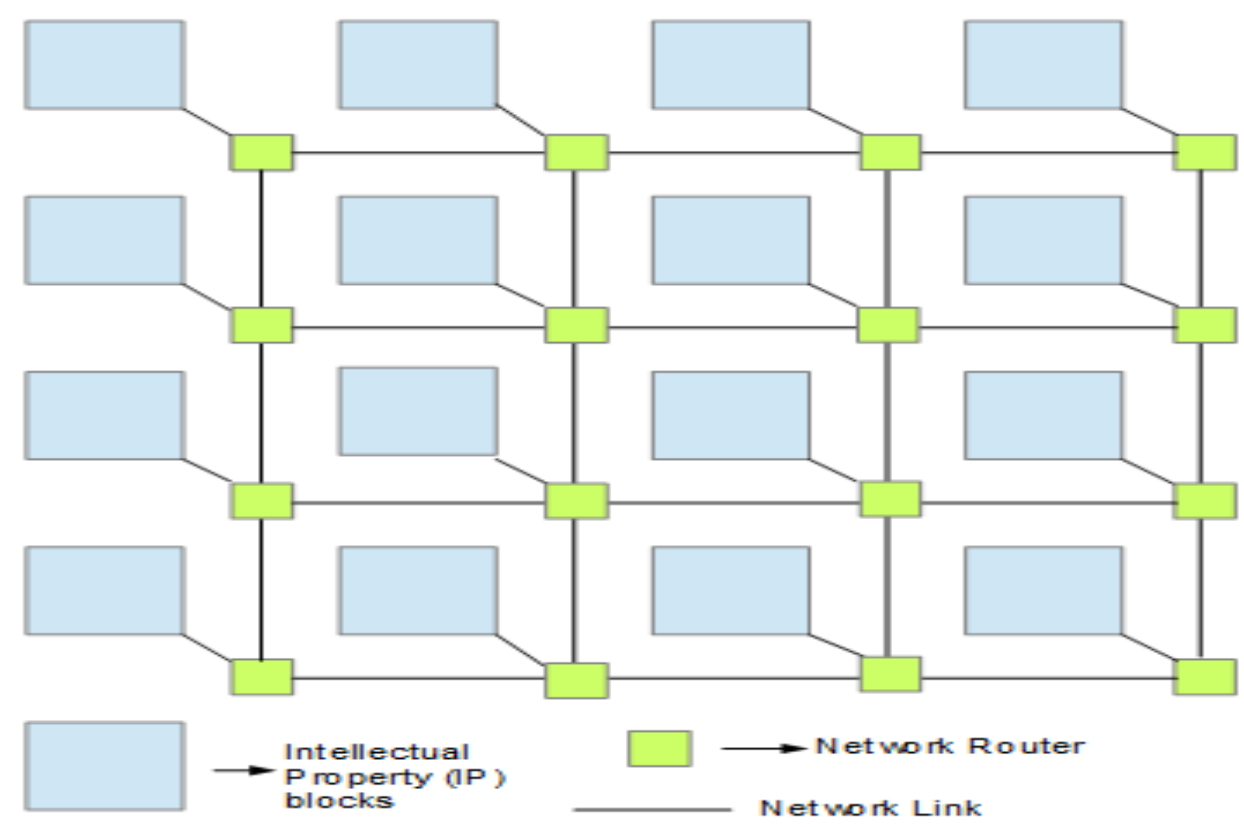

Figure 1-2: Network-on-Chip architecture 
Network on chip uses a modular approach for communication between the cores. Each core or processing element is connected to a network router and the network routers are interconnected using some network topology. SPIN, CLICHÉ or Mesh, Torus, Folded Torus, Octagon and Butterfly Fat Tree (BFT) are some of the network architectures [6]. Figure 1-2 depicts the CLICHÉ network architecture.

There are various switching techniques used for the data to reach from source to destination. Switching techniques used are: Circuit Switching, Packet Switching and Wormhole Switching. In Circuit Switching, a complete path needs to be set up between the source and the destination before the real communication begins. The whole physical link cannot be used until entire transmitted data reaches the destination. The disadvantage with this kind of switching is the set up of path is very slow which delays the transfer of message from source to destination. Moreover, the channels between source and destination are not being used during the idle period and this leads to low channel utilization [7]. In packet switching, data is divided into fixed length units called the packets. Each packet has the routing information and hence there is no need for the path set up. The network router routes the packets based on the routing information within the packet. Since each packet needs extra bits to store the routing information, the size of the buffers required at the switches is high. Moreover since each packet is routed using the routing information, the packets may reach the destination out of order and some additional processing would be required to put the message back together. In wormhole switching, each packet is divided into fixed length flow control units (flits). The header flit has the routing information and is used to establish a path from source to destination. The 
body flits follow the path established by the header flit. Since the size of the flits is small, the buffer space required at the intermediate switches would be less. Since the header flit establishes a path between source and destination, it can block other communications. This problem is solved by introducing virtual channels (VC's) in the intermediate switches as shown in figure 1-3.

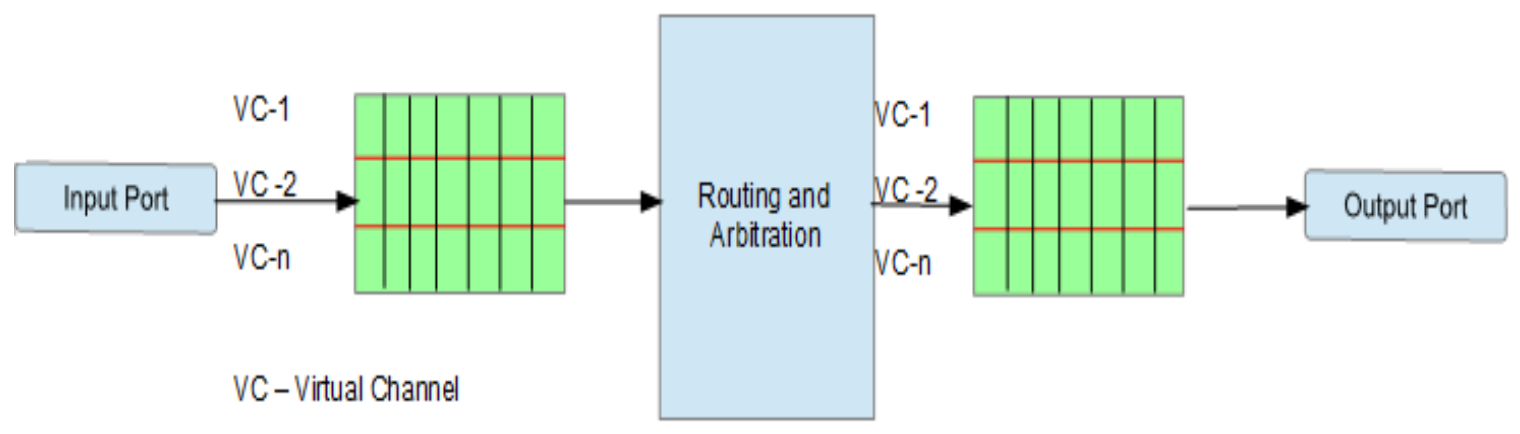

Figure 1-3: NoC switch architecture

Each incoming port and outgoing port will have multiple VC's to hold flits belonging to different packets. Arbitration will be done to use the link interconnecting the network routers. If all the VC's are full then the header flit will be dropped and the source will have to retransmit the header flit. Figure 1-3 shows the basic switch architecture.

\subsection{Emerging interconnects}

The most frequently used interconnects are electrical wires for transfer of data and control messages between the cores. However as fabrication technologies scale down, the size of electrical wires also scales down resulting in increased resistance of the wire. Moreover for shared medium arbitrated bus, each core connected to the shared bus increases the intrinsic parasitic capacitance. This increased resistance 
and intrinsic parasitic capacitance of electrical wire results in higher propagation delay and heat dissipation in case of global communication. The bandwidth offered by electrical wires is also very less. Hence multiple wires would be needed for communication between a pair of cores. Future chips are expected to have hundreds to thousands of cores. Laying down parallel buses with increase in number of cores would be impossible thus affecting the scalability of the system. According to International Technology Roadmap for Semiconductors (ITRS) novel scalable interconnects would be needed to meet the performance requirements of the future chips.

Some of the emerging interconnect paradigms are three dimensional (3-D) integration, wireless interconnects and photonic interconnects. These interconnect technologies are envisioned to support communication on a multi-core chip.

3-D integrated circuits (IC's) use 3-D integration to vertically stack dies with through silicon vias (TSV) for inter-layer communication. Various architectural designs for 3-D NoC are Symmetric NoC Router Design, 3-D NoC-Bus Hybrid Router Design, True 3-D Router Design and Multi-layer 3-D NoC Router Design [8]. The major advantage of 3-D IC's is considerable decrease in length and number of global interconnects, resulting in an increase in the performance and decrease in power consumption and area of wire limited circuits. Despite the advantages major challenges in implementing 3-D NoC are crosstalk and noise analysis, thermal mitigation and interconnect modeling [9]. 
In Wireless NoC, the global communication takes place using single hop, longrange, high bandwidth and low energy links operating in the millimeter (mm)-wave frequency range [10]. Various architectures have been proposed for reduced energy dissipation and latency designed using traditional CMOS technology. One such architectural implementation has been shown in [10]. However it has been predicted that the intra-chip communication using the bandwidth available using conventional CMOS based RF technology is not going to be sufficient [11]. Recent research has been directed towards carbon nanotubes (CNTs) since they exhibit excellent emission and absorption characteristics leading to dipole like radiation behavior making them promising for use as antennas in wireless NoC [11]. But, the failures in fabrication of CNTs are much higher than CMOS process. The electrical characteristics of the CNT are difficult to control. This leads to failure of links, negating the advantages of wireless links.

Photonic interconnects are used to carry optical data from source to destination. The data from the processing element (PE) is sent to the modulator. Modulator is basically an electro-optic device. An off-chip laser source injects light of various wavelengths into the optical waveguide. Modulator converts the received data into optical data by modulating it on one of the wavelengths of the laser source. When the optical data reaches the detector, it converts the optical data back to electrical format after which it is directed towards PE.

Optical fiber can handle multiple wavelengths form the laser source at the same time. Dense Wavelength division Multiplexing (DWDM) is used to improve the data 
bandwidth. Light travels much faster in an optical interconnect as compared to packets in planar dielectric interconnects. Hence optical interconnects facilitates high bandwidth low latency communication. Moreover the energy dissipation in photonic interconnects is also less as compared to planar dielectric interconnects.

\subsection{Thesis Contribution}

In this thesis work, we propose crossbar based photonic NoC (PNoC) architecture which dynamically allocates bandwidth between different heterogeneous components like custom logic, CPUs, GPGPUs, reconfigurable hardware and distributed memory units on a chip.

The following point summarizes the contributions made during this work.

\section{$>$ Proposed Network Architecture}

A heterogeneous PNoC architecture with dynamic bandwidth allocation called $d$-HetPNoC is proposed.

\section{$>$ Experimental Evaluations}

- Develop a cycle accurate simulator to implement the PNoC architecture with 3-stage switches namely, input, output arbitrations and routing.

- Obtain experimental results of the proposed $d$-HetPNoC architecture with crossbar based Firefly architecture with respect to the following parameters using the cycle accurate simulator

- Peak achievable bandwidth

- Packet energy dissipation

- Non-uniform traffic patterns 
- Area overheads

\section{$>$ Publication}

- Ankit Shah, Naseef Mansoor, Ben Johnstone, Amlan Ganguly, Sonia Lopez Alarcon. "Heterogeneous Photonic Network-on-chip with Dynamic Bandwidth Allocation" System on chip conference (SoCC) 2014 in Las Vegas, Nevada. 


\section{Chapter 2 Related Work}

Recent advances in the fabrication technology have made it possible to integrate various photonic elements on multi-core chips. Consequently various high performance and low energy architectures have been proposed for implementation of PNoC. The various photonic elements and architectures used in the study are shown below.

\subsection{Photonic Elements}

The on-chip laser source, Micro Ring Resonators (MRR) and the photonic waveguide are the most important components of PNoC. On-chip laser source provides the necessary multi-wavelength light source. PNoC uses Dense Wavelength Division Multiplexing (DWDM) for increasing the bandwidth of the optical fiber links. Micro Ring Resonator is used for converting the electrical packet to optical packet of particular wavelength. Photonic waveguide is used to carry the photonic packets between photonic routers. Multiple demodulators are used to filter the data packets modulated on specific wavelength. Each of the photonic elements is explained in depth in the section below.

\subsubsection{Micro ring resonator}

The micro ring resonators (MRRs) act as optical filters and can be made into electro-optical modulators, lasers and detectors when carrier injection, optical gain or absorption mechanisms are incorporated. Photonic network needs high integration density and low power consumption making micro ring resonator a popular choice because of its small size, high quality factor $Q$, transparency to off- 
resonance light and no intrinsic reflection [12]. Silicon adiabatic micro ring resonators with radius of $2 \mu \mathrm{m}$ are shown in [13]. These micro ring resonators provide high integration density because of small radius. Moreover, these MRRs consume less power as the power consumption of the modulator is directly proportional to the circumference and inversely proportional to quality factor $\mathrm{Q}$ of micro ring resonator. Also the total bandwidth of the micro-ring based WDM modulation system is limited by free-spectral range (FSR). FSR is inversely proportional to the circumference of the MRR. Hence these MRRs have FSR of 6.92 THz making it possible to fit in more wavelengths and thus increasing the total aggregate data bandwidth [13].

The MRRs can modulate the light signal from the laser source at a speed of 12.5 $\mathrm{Gb} / \mathrm{s}$. The adiabatic micro ring modulators are able to meet the requirements of the PNoC architectures by providing better power consumption and lesser resistance than the older mach-zehnder modulator (MZM) [12]. The light wave from the laser source consists of various wavelengths. Only the light wave whose frequency matches the resonant frequency of the MRR will be coupled on to the MRR. Light waves of other frequencies will not be affected. The resonant frequency of each MRR can be changed by applying heat to them. The heat is applied on the MRR with the help of local heaters. We assume a single heater element per MRR in the PNoC to enable the thermal tuning. Hence each MRR can be tuned to a different resonant frequency thus utilizing all the frequencies from the laser source and enabling WDM for higher aggregate data bandwidth. 


\subsubsection{Photo-detector}

The demodulation is done using the on-chip photo-detectors. When WDM is used, MRR is used in conjunction with on-chip photo-detector. MRR is used for selecting the light having the same wavelength as resonant wavelength of the MRR. The filtered output of the MRR goes to a germanium (Ge) p-i-n photo-detector which absorbs the light and converts it into electrical current. This current is amplified and fed to a threshold device. If the electric current is greater than the threshold voltage it is considered as 1 else it is considered as 0 . Photo-detector parameters such as power consumption, bit rate and photo-detection threshold play an important role in governing the efficiency of PNoC. Germanium photo-detectors of dimension $0.7 \mathrm{umx} 20 \mathrm{um}$ have been demonstrated to operate at $40 \mathrm{Gbps}$ [13]. The photo detector responsivity as high as 1.08A/W has been demonstrated [14].

\subsubsection{Photonic Switching Elements (PSEs)}

PSEs are required in some PNoC architectures to turn light by $90^{\circ}$. PSEs are made up of MRRs. The basic structure of PSE is shown in Figure 2-1. When the PSE is in off state the light passes through without making a turn as shown in figure 2-1 (b). When the PSE is in on state, the wavelength of light which matches the resonant wavelength of MRR gets turned by $90^{\circ}$ as shown in figure 2-1 (a). An example of PNoC requiring PSEs is the 2Dimensional Folded Torus (2DFT) [15]. This PNoC uses electronic network to carry the header flits and the photonic network to carry the body flits. Header flit of the photonic packet uses the electronic network to set up the path from source to destination using dimension order routing. Header flit 
reserves each intermediate router for the photonic flits that are supposed to follow. Since this PNoC architecture uses dimension order routing, photonic flits may need to turn at each intermediate router. This is done by means of PSE. Implementation of a blocking router using PSEs has been demonstrated in [15].

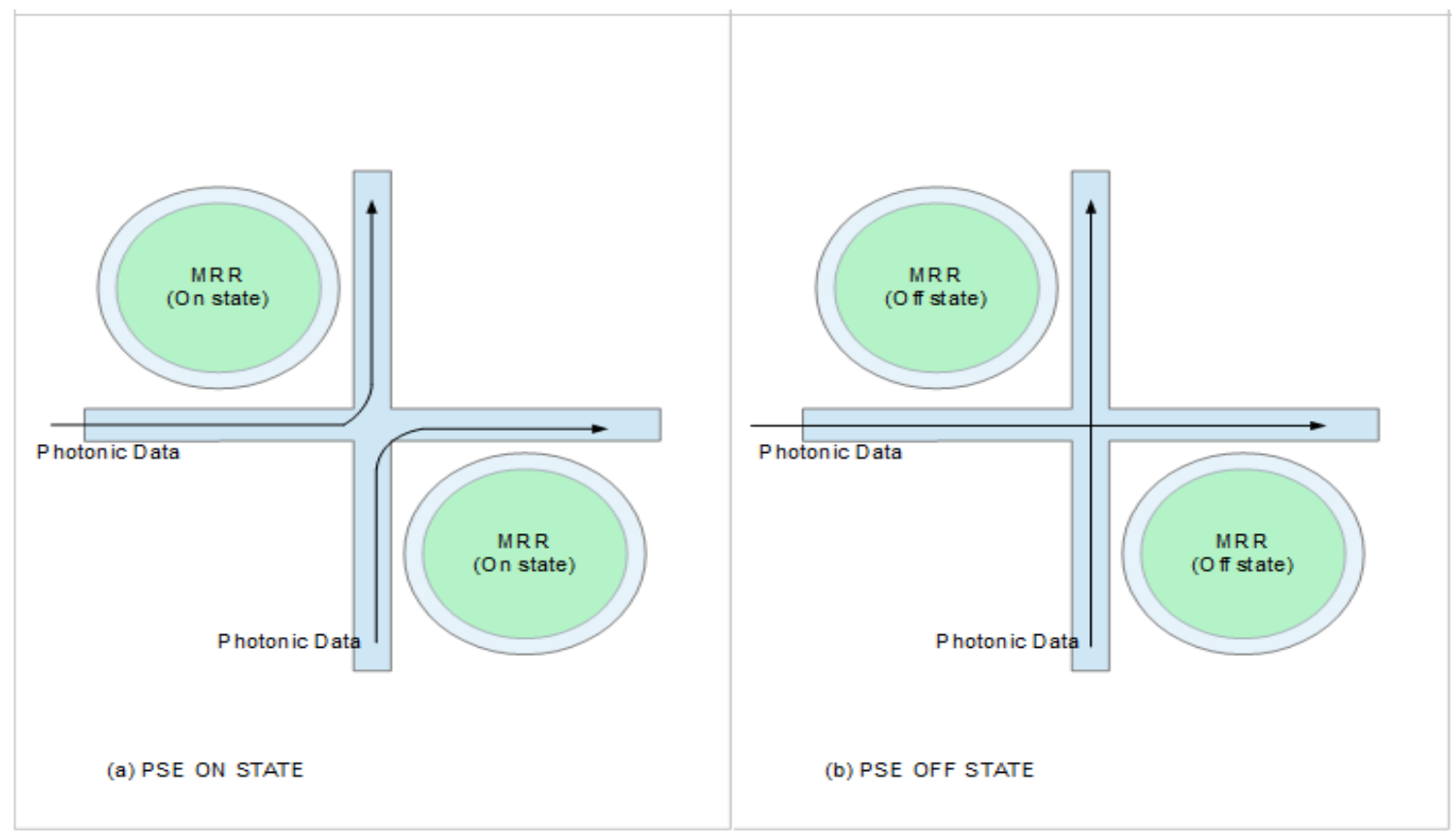

Figure 2-1: A basic photonic switch

Though a blocking router restricts simultaneous flow of information in multiple directions, constructing a non-blocking switch using PSEs requires a highly complex structure. This has a negative impact on the area and, more importantly, the optical signal integrity, as each PSE hop introduces additional loss and crosstalk. Therefore, the design choice would be to blocking switch because of its compactness and to bear it's blocking properties in mind when designing the network topology and routing algorithm [15]. 


\subsubsection{Laser Source}

A multi-wavelength laser source, with small area is required for a PNoC. There are 2 types of multi-wavelength laser source: off-chip laser source like the comb laser source which is coupled to the chip using fiber optics and on-chip laser source like single wavelength distributed-feedback (DFB) laser array [16]. It has been demonstrated in [16] that heterogeneously integrated on-chip sources are preferred as they are energy efficient and energy proportional and result in overall system efficiency.

\subsubsection{0ptical waveguides and couplers}

The on-chip optical waveguides are work on the same principle of the conventional optical fibers that carry optical signal over long distance. On-chip optical waveguide consists of core which carries the light and cladding surrounding the core. The core and cladding is made of materials with different refractive index. Refractive index of cladding is significantly lower than the refractive index of core thus causing total internal reflection of light and confining the optical signal within the core. The optical signal undergoes multiple reflections inside the core while moving along the waveguide. In PNoC, nanophotonic waveguides in silicon on insulator (SOI) fabricated with deep ultraviolet (UV) lithography is used as the medium for carrying the optical packets [17]. For low power consumption, the output from the laser diode should be efficiently coupled with the silicon waveguide. Spot-size converters (SSC) are used to couple the laser light from laser diode to silicon waveguide. The photo-detectors can be fabricated by bonding of InGaAs/InP 
wafers directly to silicon waveguides and formation of metal-semiconductor-metal structures, giving responsivities as high as $0.74 \mathrm{~A} / \mathrm{W}$ [18]. A complete optical transmission link, having a single silicon waveguide integrated with both laser diode and photo-detector, is demonstrated in [19].

\subsection{Existing PNoC Architectures}

The PNoC in existing literature that is used in the study is the Firefly Architecture [20].

\subsubsection{Firefly Architecture}

Firefly architecture is a cross-bar based hybrid, hierarchical architecture. Four processing elements share a router to form a local node. Local nodes within the cluster are connected in form of Concentrated MESH (CMESH). Communication between the nodes within the cluster takes place using the traditional electrical networks thus exploiting the benefits offered by electrical interconnects for short, local communication. Inter-cluster communication takes place using nanophotonic interconnects.

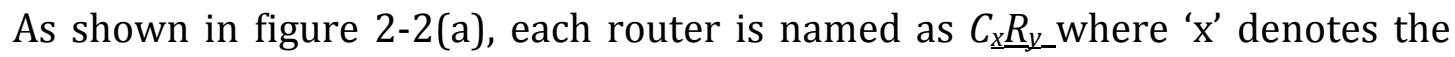
cluster number and ' $y$ ' stands for assembly number. All the routers having same ' $x$ ' value belong to same cluster and hence communicate using electrical interconnects. All the routers having the same ' $y$ ' value belong to an assembly and are interconnected using cross-bar based nanophotonic interconnects as shown in figure 2-2(b). 


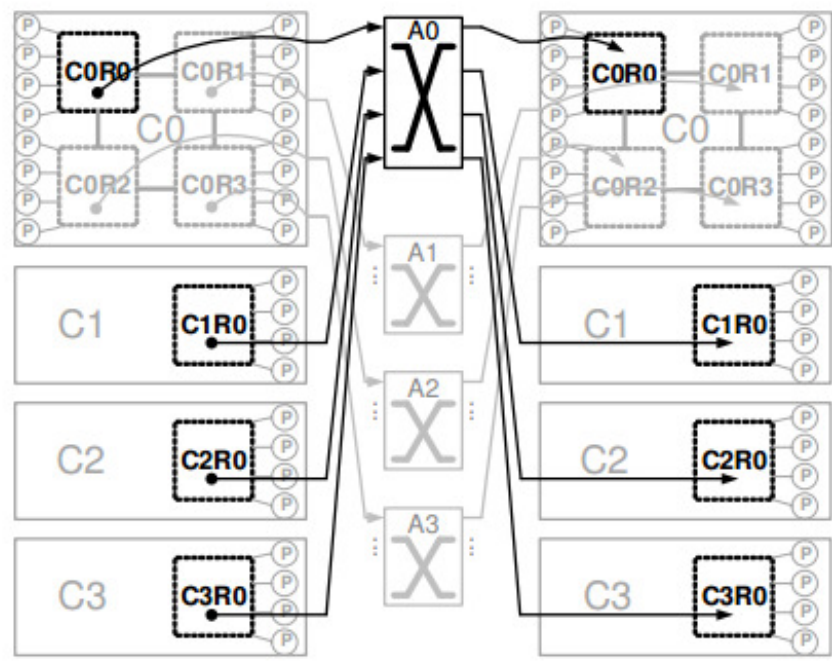

(a)

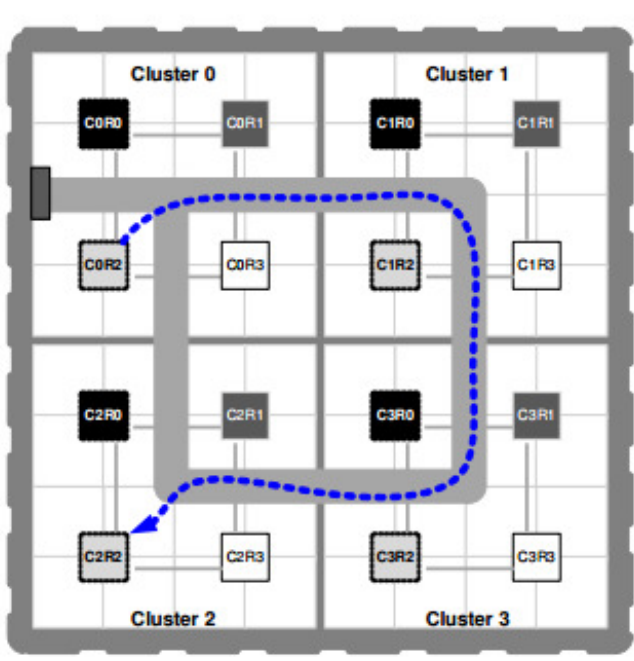

(b)

Figure 2-2: Firefly Architecture: (a) Cross-bar between clusters of same assembly, (b) waveguide supporting inter-cluster crossbars. Reproduced form [20]

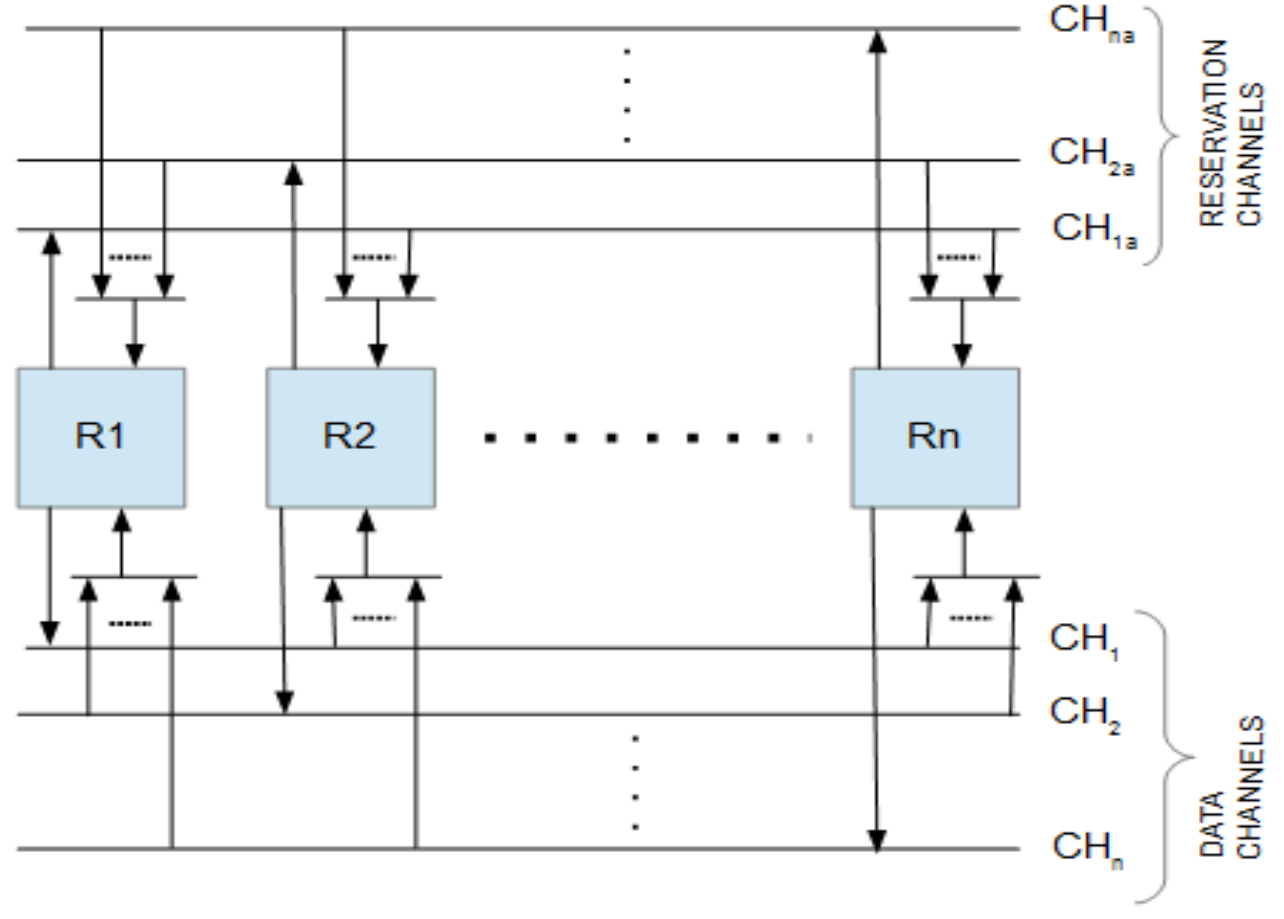

Figure 2-3: Reservation-assisted Single Write Multiple Read. Adapted from [20] 
Firefly architecture uses Reservation-assisted single write multiple read (RSWMR) for implementing nanophotonic crossbar. There are two types of channels for communication between the routers; Reservation channel and Data channel. Reservation channel is used to establish a path between source router and destination router. Data channel carries the actual data from source router to destination router. Reservation channels carry the reservation flit which contains the source router id, destination router id and duration of communication. Once reservation flit reaches the destination, the photo-detectors on the data channel corresponding to source router are turned on to accept the data. Conceptual diagram of R-SWMR is shown in figure 2-3.

The disadvantage of this architecture is that its inability to dynamically assign bandwidth between pair of nodes between clusters. Also since all the modulators and demodulators are on for any communication, this architecture is energy inefficient. 


\section{Chapter 3 Dynamic Heterogeneous Photonic NOC ( $d$-HetPNoC)}

Bandwidth requirement between various cores in future multi-core chips will dynamically vary depending on the mapped applications and nature of the cores. The applications mapped on specific cores may change over time due to various reasons such as start and end of a task or dynamic thermal management schemes such as temperature-aware task allocation. This will result in dynamically varying traffic patterns between the cores. In order to cater to such dynamically varying demands of bandwidth between communicating pairs we propose a scheme to allocate bandwidth-on-demand for photonic NoC architectures. Recent literature has explored photonic NoCs from tile based architectures to crossbar based high radix ones [15], [20] - [22]. It is argued in [23] that crossbar-based photonic NoC architectures can scale better in terms of reliability and performance by using novel photonic devices with crosstalk suppression. Hence we modify a crossbar based baseline photonic NoC architecture to enable the dynamic bandwidth allocation.

\subsection{Network Architecture}

The crossbar architecture adopted is a Single Write Multiple Read (SWMR) photonic crossbar. Cores are grouped in clusters and each cluster will have a data channel consisting of multiple DWDM wavelengths to all other clusters. An energyefficient variation of the SWMR crossbar has been demonstrated in [20], where a reservation request is broadcast on separate channels from the source cluster to establish a path containing the destination ID. This allows the destination to keep the 
demodulators to be switched on only when it receives a packet rather than always, thus saving energy. We propose to modify this baseline crossbar so that in addition to establishing a path, a variable number of wavelengths are allocated to the channel in proportion to the traffic requirement. This traffic requirement is determined by the task running on the cores, which governs the frequency and volume of data communication with other cores. Consequently, this bandwidth allocation happens whenever there is a change in the task mapping on the chip and not on a per-packet basis. Hence, the overheads associated with this scheme are greatly mitigated.

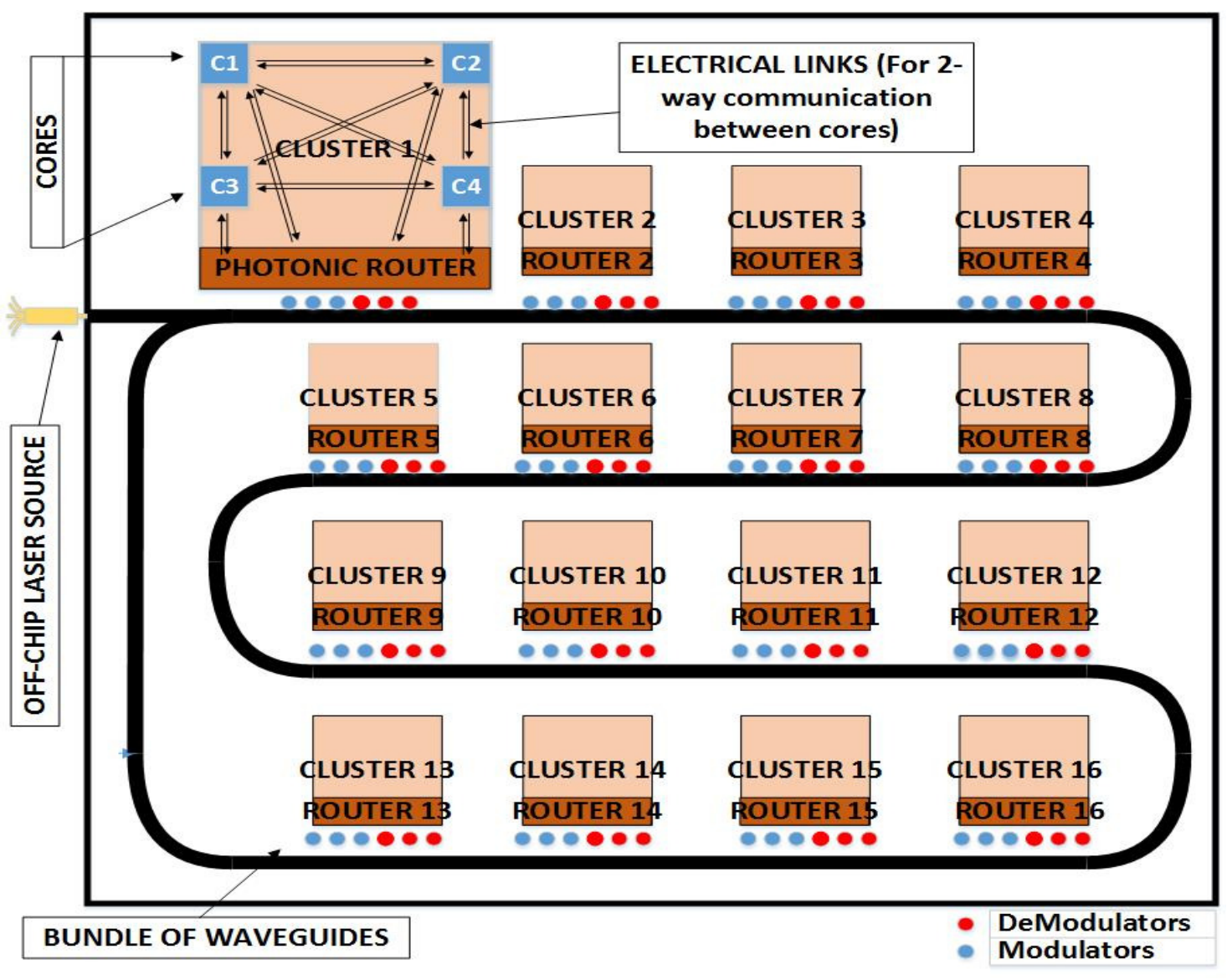

Figure 3-1: Dynamic bandwidth allocation enabled PNoC architecture.

In the proposed $d$-HetPNoC, we have considered a hierarchical, hybrid configuration crossbar as in [20]. The whole CMP is divided into clusters of 4 cores. 
These 4 cores are interconnected using traditional copper interconnects in an all-toall manner avoiding multi-hop paths within a cluster. As the cores in a cluster are physically close, using wire line links can achieve reliable and fast communication. This intra-cluster configuration is different from the concentrated Mesh in [20]. Each cluster is equipped with a photonic router, which is interconnected using photonic channels with all other photonic routers. This architecture is shown in figure 3-1.

\subsection{Dynamic Bandwidth Allocation (DBA) Mechanism}

DBA is possible by assigning variable number of wavelengths to the write channels of the clusters. When there is a change in the task allocation on a core, the network reconfigures itself and allocates necessary bandwidth to the cluster of the core. The total aggregate data bandwidth depends on the total number of DWDM wavelengths in all the data waveguides together. Since this aggregate bandwidth budget has to be shared between all the clusters we propose a token-based distributed mechanism to request and acquire wavelength channels in each photonic router.

\subsubsection{Token Passing based Channel Allocation}

The DBA is achieved by using a token-based mechanism. This mechanism grants the right to request bandwidth or wavelengths to one photonic cluster at a time to avoid reusing already allocated wavelengths within a single waveguide. This token is circulated between the photonic routers using a separate control waveguide with maximum DWDM. The token consists of several bits where, each bit in the token denotes the status of a specific wavelength in a specific data waveguide i.e., whether 
it is currently allocated to any router or not. The size of the token in bits, $N_{T W}$ is equal to the total number of wavelengths, which can be dynamically allocated and given by,

$$
N_{T W}=\left(\lambda_{W} * N_{W}\right)-N_{\lambda R}
$$

In (1), $N_{W}$ is the number of waveguides needed for data communication, $\lambda_{W}$ is the number of DWDM wavelengths that can be accommodated in a single waveguide and $N_{\lambda R}$ is sum total of the number of wavelengths reserved by each cluster as discussed later in this section. When a cluster has the token, the photonic router can acquire wavelengths and change their status based on its requirements.

If there is any change in the applications running on a particular core, it sends an updated demand for bandwidth to the photonic router. This information is in the form of a demand table, which contains the number of wavelengths required for communication with all the other clusters. We have assumed that the core will determine these numbers based on the traffic requirements of the current task with all other clusters. The photonic router consists of 6 tables; current table, request table and 4 demand tables from the 4 cores. The current table consists of current bandwidth allocated to the cluster for communication with the other clusters. This table is initialized to a certain predetermined minimum number such that each cluster has a minimum bandwidth allocated to its write channel. This ensures that no cluster starves even if all other clusters consume all the data bandwidth. This minimum number can be determined based on the overall data bandwidth in the PNoC and is at least 1 wavelength per cluster. The total number of such reserved wavelengths for minimum bandwidth allocation is denoted by $N_{\lambda R}$ in (1). 
Each entry in the request table is the maximum of all the corresponding entries in the demand tables. In this way, the entries in the request table always contain the highest demanded bandwidths or number of wavelengths to the other clusters. Once, the photonic router acquires the token it captures or relinquishes wavelengths based on the request table and number of currently acquired and available wavelengths. The cluster aims to acquire the highest number of wavelengths among all the entries in the request table, which corresponds to the maximum bandwidth that the cluster will need for communication. Multiple wavelengths for a particular cluster could be spread over multiple waveguides depending upon availability of wavelengths. Once, the wavelengths are acquired or relinquished the current table in the router is updated to reflect the current allocated bandwidths to all other clusters. The router also records the specific identifiers of all the wavelengths it has acquired. The wavelength identifiers consist of the waveguide number and the wavelength number within that waveguide. After this the token is modified to reflect the latest status of the wavelengths and released to the next cluster.

Depending upon the availability of the wavelengths it may not be possible to satisfy all the requests from all the clusters. Hence, the request table is not modified after the wavelengths are allocated and the current table is updated. This will enable the router to try to acquire additional wavelengths if necessary the next time the token returns to the cluster. This scheme works even when the task allocation to specific cores happen asynchronously with the circulation of the token as the request table can be updated even when the token is not present in the photonic router. The micro-architecture of the photonic router is shown in figure 3-2. 
There is an overhead associated with token passing. The time taken by a token to traverse the link, $T_{L}$ between two photonic routers is given by,

$$
T_{L}=N_{T W} /\left(\lambda_{W} * B\right)
$$

Where, $B$ is the bandwidth per DWDM wavelength. The worst-case time required by a particular photonic router to repossess the token is given by $T_{L}{ }^{*} N_{P R}$, where $N_{P R}$ is the total number of photonic routers. As there is one photonic router per cluster of 4 cores, $N_{P R}$ is equal to $\left(N_{C} / 4\right)$ where $N_{C}$ is the total number of cores on the chip. In addition, there would be an overhead required by the photonic router to process demands and update the request and current tables. However, since this will happen only when there is a change in the task mapping on a core, the overheads will be greatly mitigated if not completely amortized, as these changes will happen at a slower rate by several orders compared to packet transfer. The transmission of demand tables and computation of the request tables can happen while the router is waiting to capture the token resulting in complete masking of the overhead. The updating of the request table and current table are also disjoint from the path of data flow within the router thus eliminating its impact on the data latency.

\subsection{Flow Control and Routing}

The routing and flow control is achieved by using the reservation channel assisted SWMR channels as proposed in [20]. Intra-cluster communication happens through the electronic links between the cores or from cores to the photonic router. Inter-cluster communication utilizes the photonic channels between the photonic routers. 


\subsubsection{Photonic Flow Control}

Whenever a cluster needs to communicate a packet to another cluster it broadcasts a reservation flit over its reservation channel for establishing a connection between source and destination. The reservation flit contains the ID of the destination, and the wavelength identifiers that are to be used for this pair from the current table at the source. The specific wavelengths are chosen among the allocated ones for the cluster based on the corresponding entry in the demand table for the destination. Upon receiving the wavelength identifiers, the destination cluster switches on the demodulators on those specific wavelengths only for the duration of a packet. This results in energy savings compared to Firefly where all the wavelengths are turned on for all transmissions irrespective of the required data rate. It will be shown in section 4.1 that the timing requirement of piggybacking the wavelength identifiers with the reservation flit in $d$-HetPNoC results in no additional timing overheads.

\subsubsection{Photonic Router Architecture}

All the intra-cluster routers are electronic which are responsible for packet transfer between cores within a particular cluster. These are 3-stage routers with input arbitration, routing/crossbar and output arbitration adopted from [24]. The photonic router in each cluster has a similar micro-architecture as the electronic routers. They have 4 electronic links to the 4 switches in its cluster and photonic channels to other clusters. The photonic router with DBA is schematically shown in figure 3-2. 


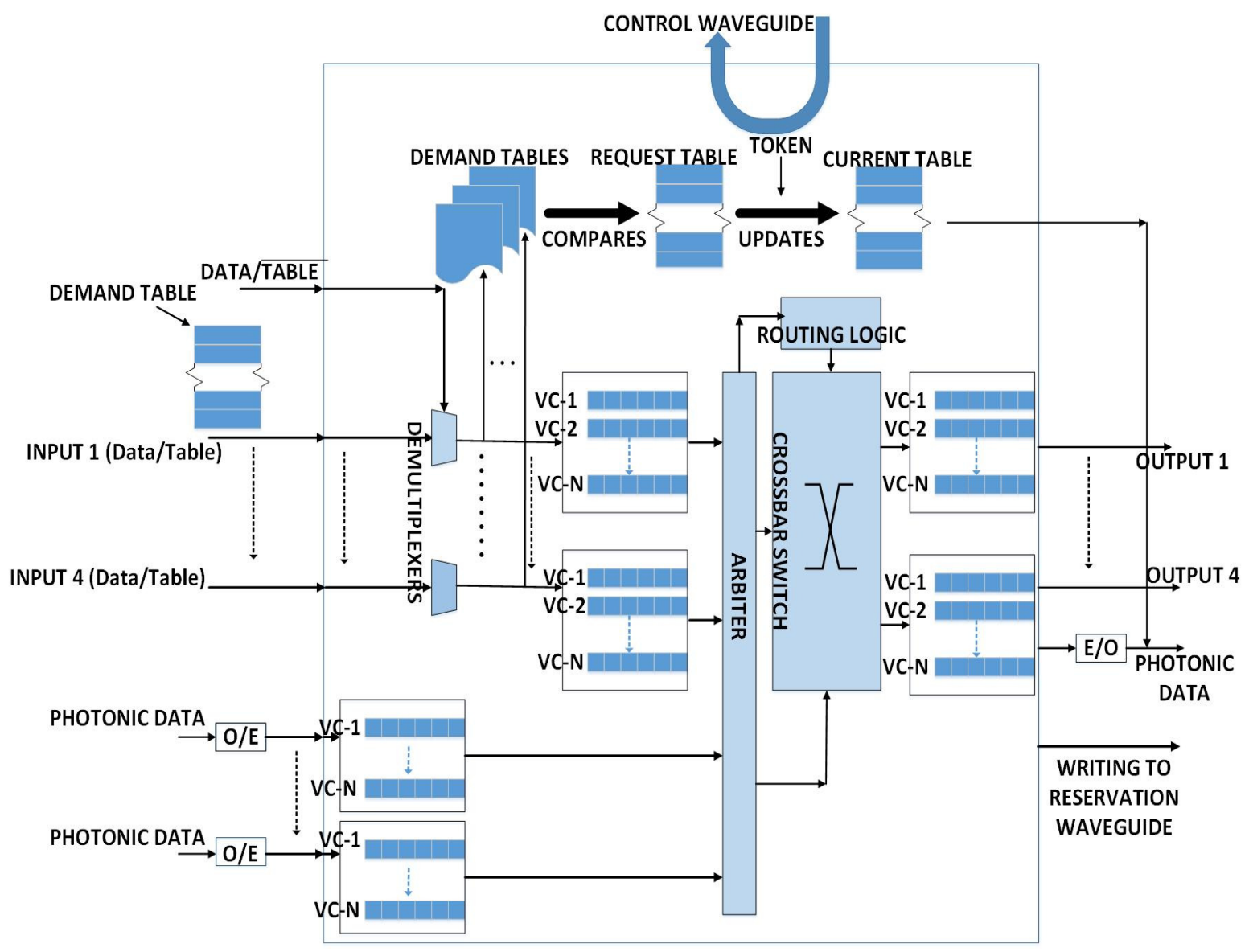

Figure 3-2: Microarchitecture of photonic router.

Using this fabric of hybrid and hierarchical photonic crossbar based NoC architecture with non-uniform DBA, we improve performance of the CMPs, which are designed for applications with heterogeneous and dynamically varying traffic patterns. In the next section, we present experimental evaluation of the proposed architecture. 


\subsection{Experimental Results}

In this section, we evaluate the performance and energy efficiency of the proposed $d$-HetPNoC architecture and compare it with the baseline crossbar-based Firefly architecture. Traffic patterns that require uniform bandwidth as well as highly unbalanced bandwidths are used to evaluate these architectures.

\subsubsection{Performance Evaluation of the $d$-HetPNoC}

Applications mapped on the cores can demand high or low bandwidths with other clusters. For our experiments we have considered 3 sets of 4 different bandwidths for the photonic channels. Different sets of bandwidths used for communication between different cores are shown in table 3-1.

\begin{tabular}{|c|c|c|c|c|}
\hline Bandwidth(BW) Set & \multicolumn{4}{|c|}{ Bandwidth (Gbps) } \\
\hline BW Set 1 (Total Wavelengths $=$ 64) & 12.5 & 25 & 50 & 100 \\
\hline BW Set 2 (Total Wavelengths $=$ 256) & 50 & 100 & 200 & 400 \\
\hline BW Set 3 (Total Wavelengths $=512$ ) & 100 & 200 & 400 & 800 \\
\hline
\end{tabular}

Table 3-1: Frequency of communication for applications with different bandwidth for skewed traffic scenarios

\begin{tabular}{cccccc}
\hline \multirow{5}{*}{ Application } & $\mathbf{1 0 0}$ & $\mathbf{5 0}$ & $\mathbf{2 5}$ & $\mathbf{1 2 . 5}$ & \\
& Gbps & Gbps & Gbps & Gbps & \\
\hline & $50 \%$ & $25 \%$ & $12.5 \%$ & $12.5 \%$ & Skewed1 \\
& $75 \%$ & $12.5 \%$ & $6.25 \%$ & $6.25 \%$ & Skewed 2 \\
& $90 \%$ & $5 \%$ & $2.5 \%$ & $2.5 \%$ & Skewed 3 \\
\hline
\end{tabular}

Table 3-2: Frequency of communication for applications with bandwidth set 1 for skewed traffic scenarios 
Electro-optic modulators and demodulators operating at $12.5 \mathrm{Gbps}$ on a single wavelength carrier channel have been demonstrated [28]. Hence, the minimum channel bandwidth we have considered is $12.5 \mathrm{Gbps}$, which can be realized with a single wavelength. Higher speed channels can be achieved by using higher number of wavelengths. The number of wavelengths required by an application running on a core is given by dividing the required bandwidth by minimum channel bandwidth. The values shown in table 3-2 represent the actual memory-interaction bandwidths required by various processing cores (e.g. CPU, GPGPU, custom logic etc.) [2].

We experimented by applying the skewed traffic patterns in table 3-2 to the different bandwidth sets in table 3-1 to study its effect on throughput and energy per message (EPM). Traffic patterns with increasing skew demands a higher frequency of communication for high bandwidth applications over the low bandwidth ones. We also evaluate the DBA enabled $d$-HetPNoC with a uniformrandom traffic pattern where all communication requires the same uniform bandwidth and all cores communicate with all other cores with equal data rate. The performance and energy consumption of the $d$-HetPNoC is compared with that of the baseline Firefly architecture to demonstrate the advantages over a uniform bandwidth allocation.

The NoC architectures are characterized using a cycle accurate simulator that models the progress of the data flits accurately per clock cycle accounting for those flits that reach the destination as well as those that are dropped. The simulation parameters are listed in table 3-3. 


\begin{tabular}{|c|c|}
\hline \multirow[t]{3}{*}{ System Size } & Number of cores, 64 \\
\hline & Number of clusters, 16 \\
\hline & Cluster size, 4 cores \\
\hline Die Area & $20 * 20 \mathrm{~nm}$ \\
\hline Clock Frequency & $2.5 \mathrm{GHz}$ \\
\hline Simulation Cycle & 10000 with 1000 reset cycle \\
\hline \multirow[t]{3}{*}{ Packet Property } & BW Set 1: Packet Size 64 flits, Flit Size 32 bits \\
\hline & BW Set 2: Packet Size 16 flits, Flit Size 128 bits \\
\hline & BW Set 3: Packet Size 8 flits, Flit Size 256 bits \\
\hline \multirow[t]{2}{*}{ Router Memory } & VC per port, 16 \\
\hline & Buffer Depth per VC, 64 flits \\
\hline Switching & Wormhole based packet switching \\
\hline \multirow[t]{3}{*}{ Photonic Data and Bandwidth } & $\begin{array}{l}\text { BW Set } 1 \\
\text { Firefly PNOC, } 4 \text { wavelengths per channel * } 16 \text { channels } \\
\text { d-HetPNoC, maximum channel bandwidth of } 8 \text { channels }\end{array}$ \\
\hline & $\begin{array}{l}\text { BW Set } 2 \\
\text { Firefly PNOC, } 16 \text { wavelengths per channel * } 16 \text { channels } \\
\begin{array}{l}\text {-HetPNoC, maximum channel bandwidth of } 32 \\
\text { channels }\end{array}\end{array}$ \\
\hline & $\begin{array}{l}\text { BW Set } 3 \\
\text { Firefly PNOC, } 32 \text { wavelengths per channel *16 channels } \\
\begin{array}{l}\text {-HetPNoC, maximum channel bandwidth of } 64 \\
\text { channels }\end{array}\end{array}$ \\
\hline
\end{tabular}

\section{Table 3-3: Simulation Parameters}

The network switches are synthesized from a RTL level design using $65 \mathrm{~nm}$ standard cell libraries from [29], using Synopsys. The delays and energy dissipation on the wired links were obtained through Cadence simulations taking into account the specific lengths of each link based on the established connections following the topology of the NoCs.

\begin{tabular}{|l|l|}
\hline Component & Power/Energy \\
\hline Modulator/Demodulator & $40 \mathrm{fJ} / \mathrm{bit}[28]$ \\
\hline Tuning & $2.4 \mathrm{~mW} / \mathrm{nm}[28]$ \\
\hline Laser Source & $1.5 \mathrm{~mW} /$ wavelength [30] \\
\hline
\end{tabular}

Table 3-4: Power or Energy dissipation of photonic components 
The power dissipation of the photonic components such as modulators, demodulators and laser sources are as shown in table 3-4. Power dissipation is explained in detail in section 3.4.1.2. The maximum number of wavelengths that can be accommodated in a single waveguide is considered to be 64 as in [20].

\subsubsection{Peak Bandwidth}

Peak bandwidth is measured as average number of bits successfully arriving at all cores per second.

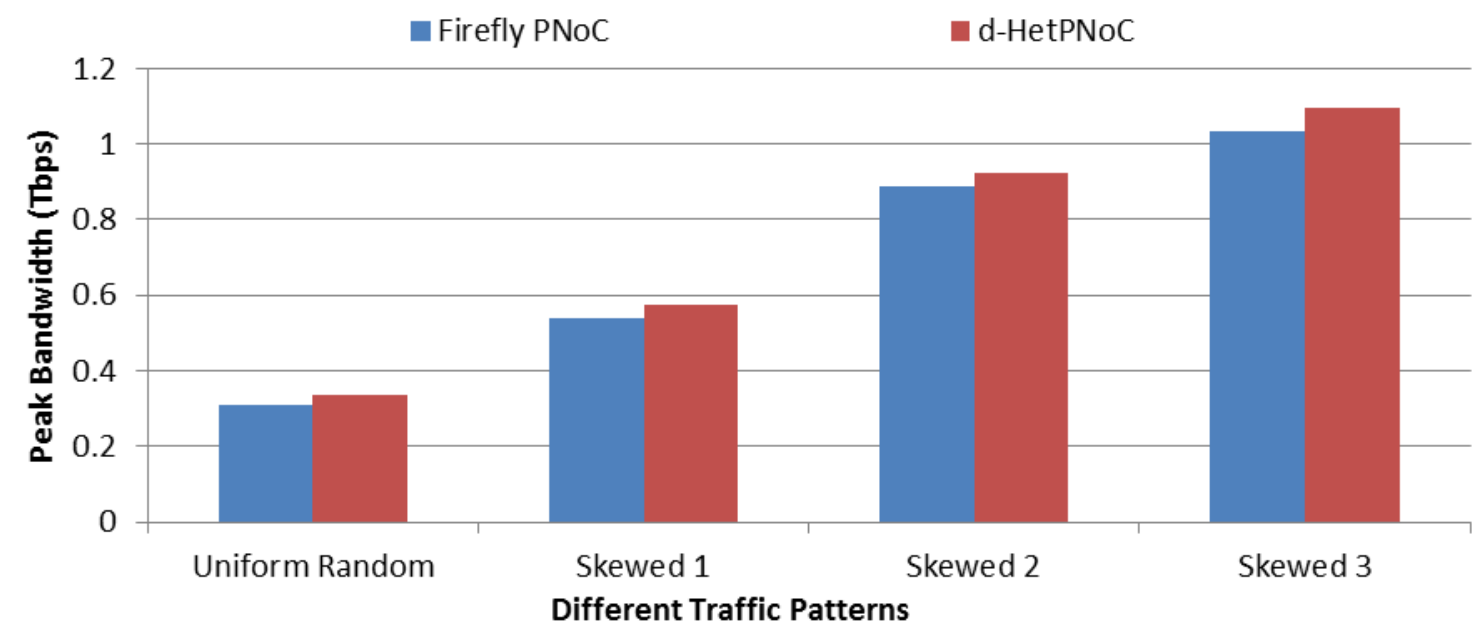

(a)

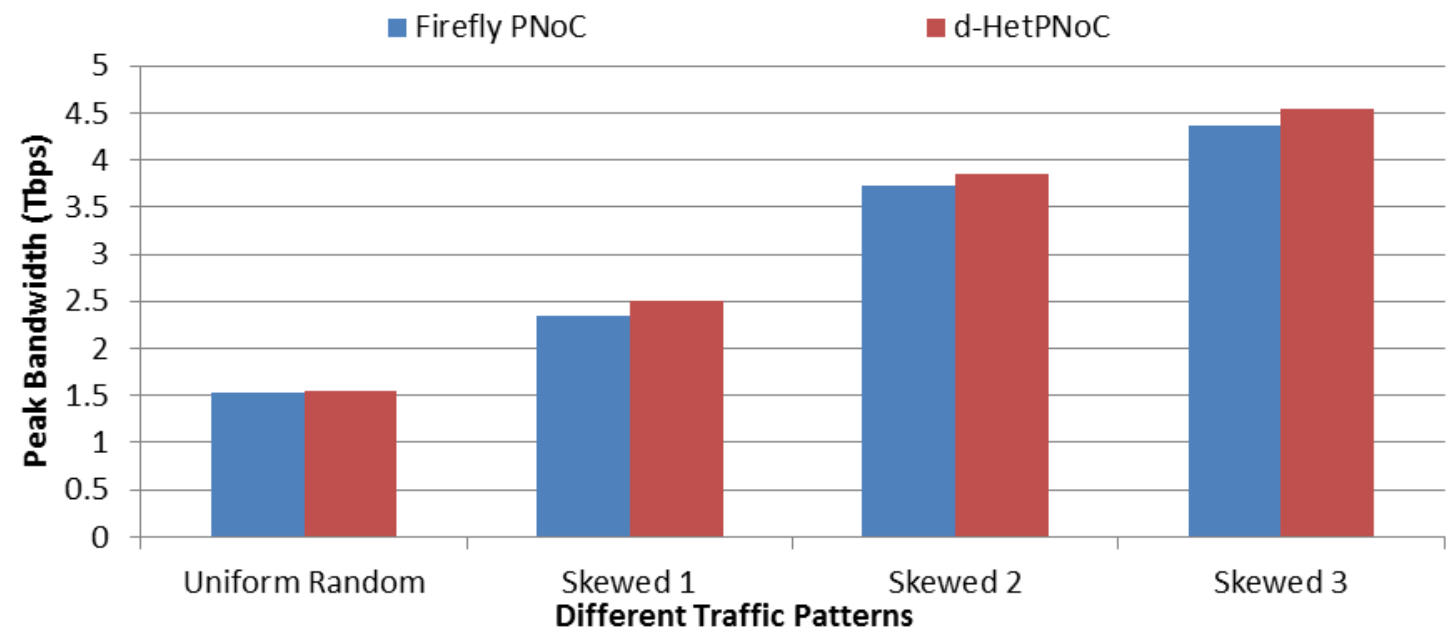

(b) 


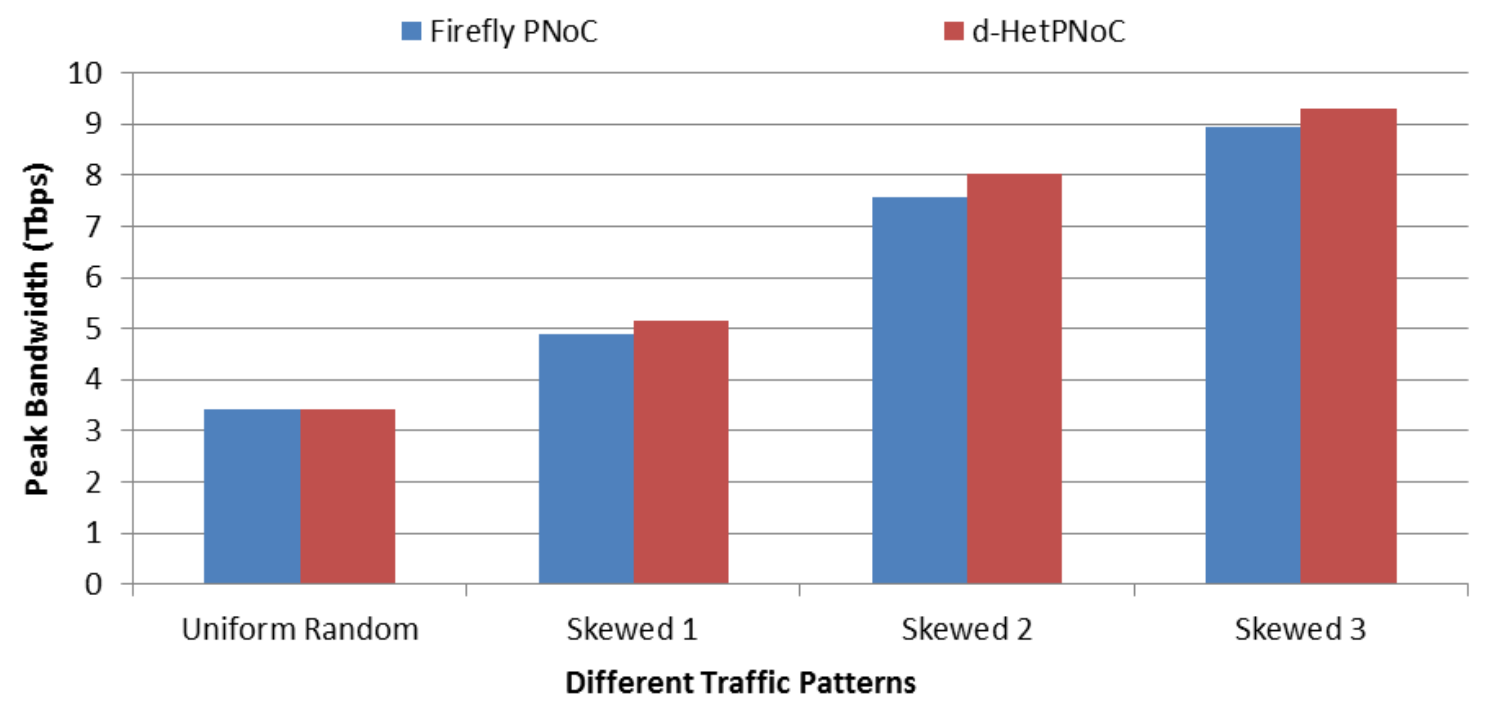

(c)

Figure 3-3: Peak Bandwidth of Firefly PNoC and $d$-HetPNoC for uniformrandom and skewed traffic patterns for (a) Bandwidth set 1 (Total Wavelengths $=64$ ) (b) Bandwidth set 2 (Total Wavelengths $=256$ ) (c) Bandwidth set 3 (Total Wavelengths = 512).

The peak bandwidth of both Firefly and $d$-HetPNoC architectures for different bandwidth sets and different traffic patterns is shown in Figure 3-3. As can be seen, with uniform traffic the $d$-HetPNoC and the baseline crossbar-based Firefly performs similarly for all three bandwidth sets as both architectures provide the exact same bandwidth between all pairs of clusters. This is because in a uniformrandom traffic all communication channels require the same bandwidth resulting in the same configuration for both Firefly and the $d$-HetPNoC. This equality is despite the fact that the $d$-HetPNoC has to send some additional information regarding which wavelengths to use to the destinations in the reservation flit along with the destination ID and packet size. The size of each wavelength identifier is 6 bits, which denote the binary encoded wavelength number (out of 64 per waveguide). For BW 
set 1 , which is the best case, a waveguide number is not needed, as a single waveguide is sufficient to accommodate all 64 wavelengths for the data channels used in our experiments. Since a cluster may need a maximum of 8 wavelengths identifiers to be sent to the destination it will take 60 ps using a single waveguide (64 wavelengths providing $800 \mathrm{Gbps}$ ) to send the reservation flit. Consequently, this information can be sent in a single clock cycle (400ps) along with the rest of reservation flit as in Firefly requiring no additional timing overhead. For BW set 3, which is the worst case, 8 waveguides are needed to accommodate 512 wavelengths. Consequently, 3 bits $\left(\log _{2} 8\right)$ would be required for waveguide number. These 3 bits denote the waveguide number in binary format. Since a cluster may need a maximum of 64 wavelengths identifiers to be sent to the destination it will take 720 ps using a single waveguide (64 wavelengths providing $800 \mathrm{Gbps}$ ) to send the reservation flit. This information can be sent in a two clock cycles along with the rest of reservation flit resulting in slightly additional timing overhead.

As the skew in traffic increases, the communication between the high bandwidth applications increases. In Firefly architecture, the uniformly assigned bandwidth is insufficient for the high bandwidth applications. This insufficient bandwidth causes the packets from this frequently communicating high bandwidth application to wait longer in the photonic routers. This in turn, congests the photonic routers resulting in a degraded performance. Conversely, the $d$-HetPNoC provides sufficient bandwidth to these high bandwidth application, reduces the waiting time for the packets in the photonic routers. Hence even with an increase in the frequency of communication between the high bandwidth applications the photonic routers do 
not suffer from congestion as much as in the case of Firefly. Consequently, the $d$ HetPNoC architecture performs better than the Firefly architecture with an increased skew in the traffic.

Percent increase in peak bandwidth of $d$-HetPNoC architecture as compared to Firefly architecture goes from as low as $0.1 \%$ in case of Uniform Random traffic to as high as $7 \%$ in case of skewed traffic pattern.

\subsubsection{Packet Energy}

There are several components of packet energy dissipation as data is transferred over the PNoC fabrics. The energy dissipated in a PNoC is given by equation(3),

$$
E_{\text {packet }}=E_{\text {electrical }}+E_{\text {photonic }}
$$

Energy dissipated by the photonic components is given by equation (4),

$$
E_{\text {photonic }}=E_{\text {launch }}+E_{\text {modulation }}+E_{\text {tuning }}+E_{\text {buffer }}
$$

where, $\mathrm{E}_{\text {aunch, }}, \mathrm{E}_{\text {moduation, }} \mathrm{E}_{\text {tuning, }}$ and $\mathrm{E}_{\text {buffer }}$ are the energy dissipated at launching photonic signals from light source, modulation/demodulation, tuning of MRR, and storing in buffer respectively. The energy dissipation per bit for various components of a $\mathrm{PNoC}$ is given in table 3-5.

\begin{tabular}{|l|l|}
\hline Component & Energy in Pico joule (pJ)/bit \\
\hline Emodulation $_{\text {E }}$ & 0.04 \\
\hline Etuning & 0.24 \\
\hline Elaunch $_{\text {buffer }}$ & 0.15 \\
\hline Erouter & 0.0781250 \\
\hline
\end{tabular}

Table 3-5: Energy of different photonic components 


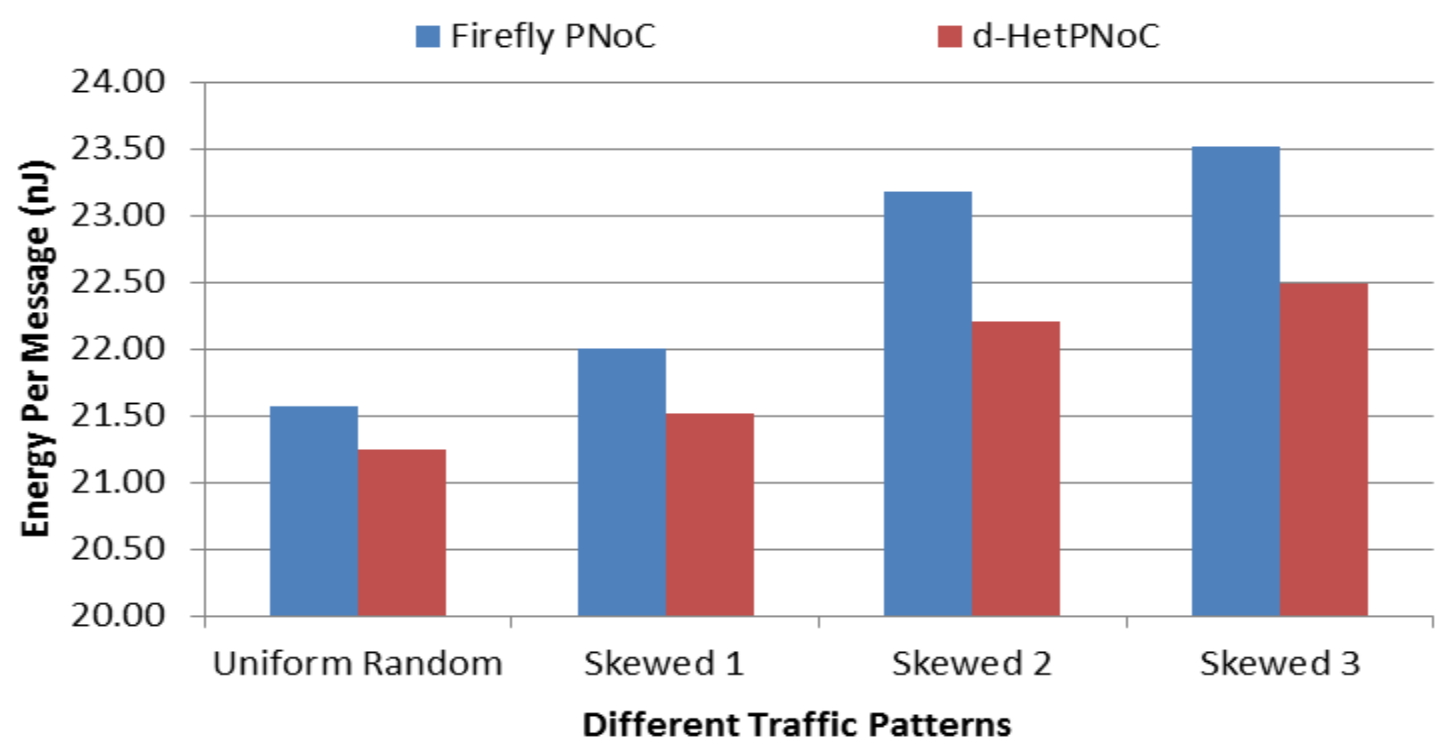

(a)

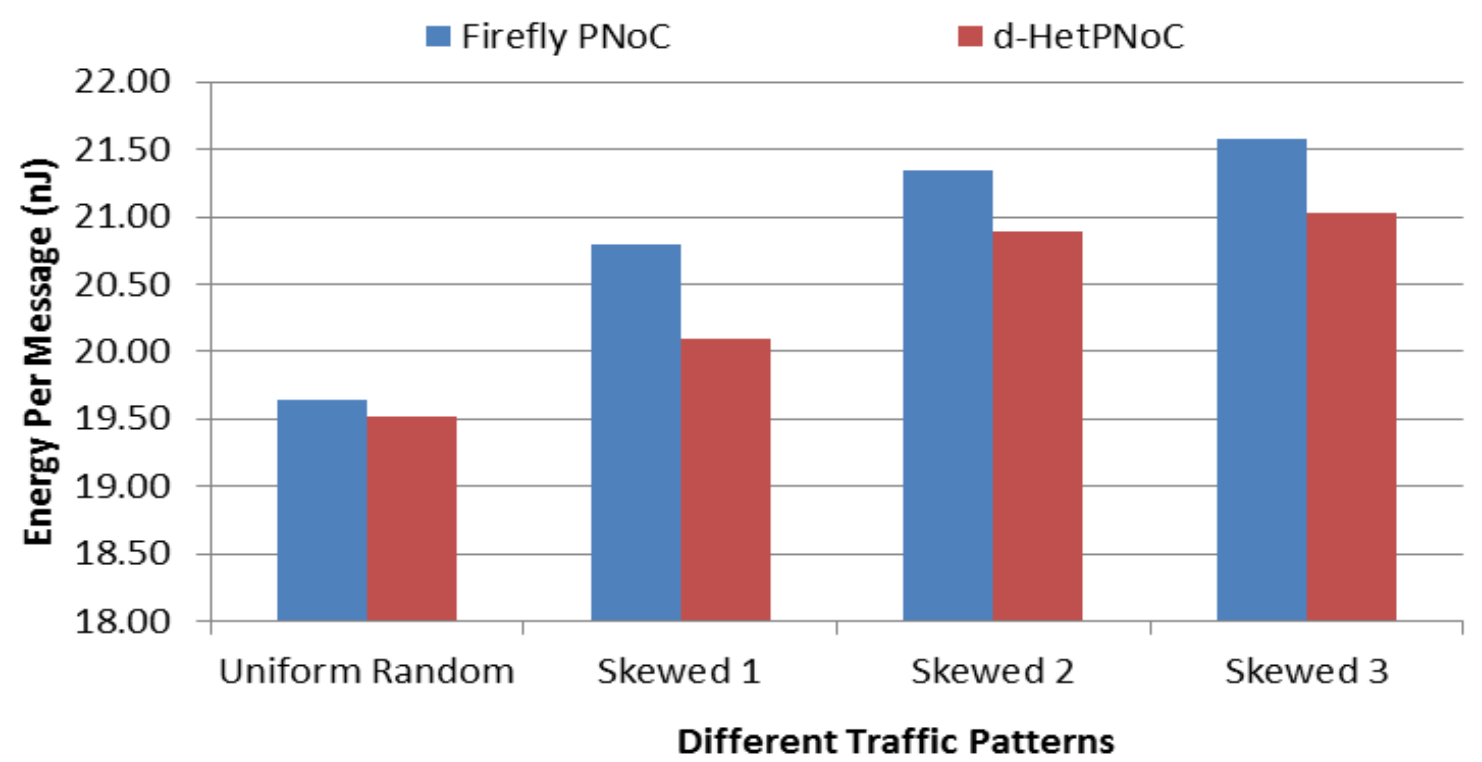

(b) 


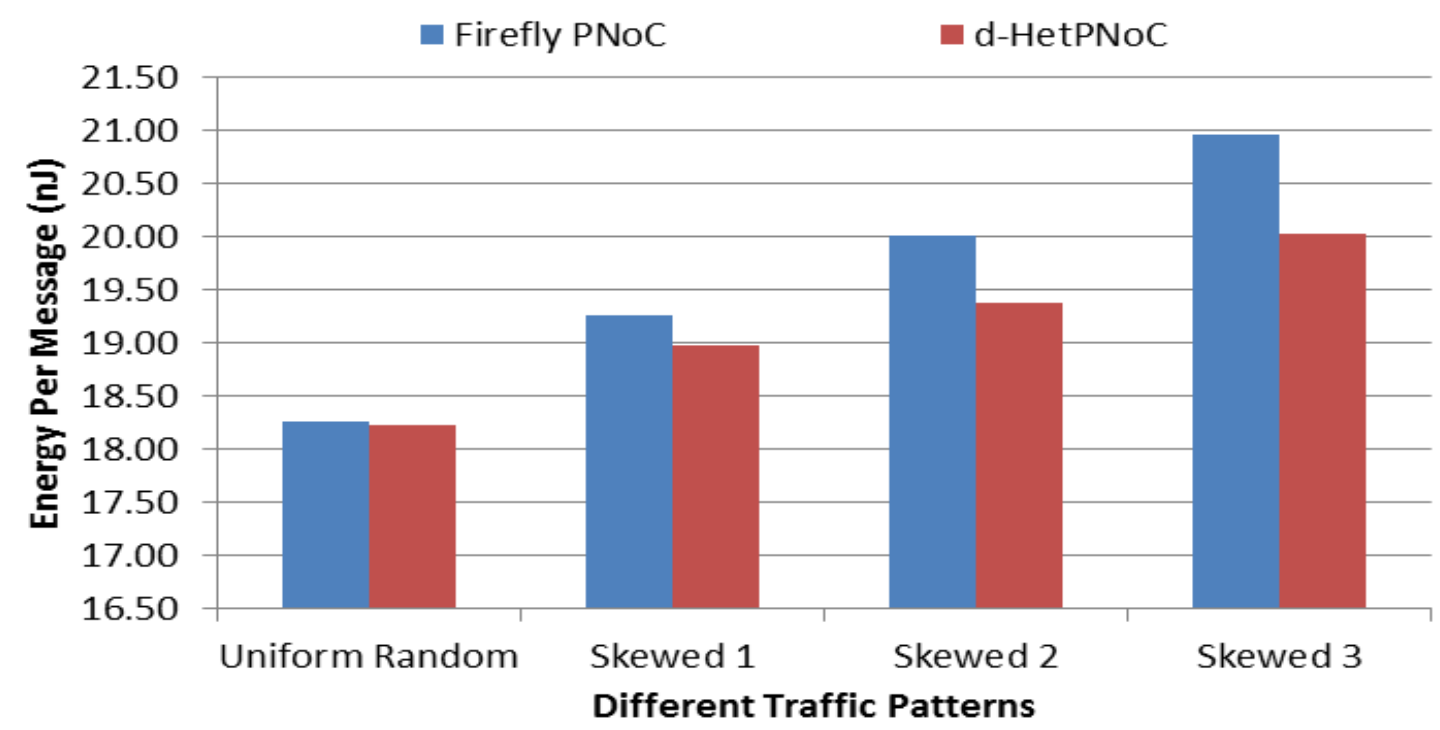

(c)

Figure 3-4: Packet Energy of Firefly PNoC and $d$-HetPNoC for uniformrandom and skewed traffic patterns for (a) BW Set 1 (Total Wavelengths = 64) (b) BW set 2 (Total Wavelengths = 256) (c) BW Set 3 (Total Wavelengths $=\mathbf{5 1 2}$ )

The packet energy of the Firefly and the $d$-HetPNoC architectures is shown in Figure 3-4. Packet energy is the energy dissipated in transferring one packet completely from source to destination at network saturation. The Firefly architecture has the same packet energy compared to the $d$-HetPNoC for the uniform-random traffic, as they are practically the same architecture in this case. However, with increased skew in traffic the packet energy also increases as the congestion in the photonic routers increases. Alternatively, for the $d$-hetPNoC the packet energy increases less with increase in skew of the traffic because of more efficient utilization of the available bandwidth. In the next subsection, we evaluate our proposed $d$-HetPNoC with specific case studies. 


\subsubsection{Case Studies with Synthetic and Real Application based traffic patterns}

In this section, we present case studies for both the architectures with synthetic and real application based traffic patterns. For the synthetic traffic patterns we considered hotspot traffic coupled with the skewed communication pattern. In this case, a core is determined to be the hotspot core and all cores send a certain percentage of all traffic to the hotspot. The rest of the traffic is distributed following the skewed traffic types outlined in table 3-1. For our case study, the skewed hotspot 1 and skewed hotspot 2 traffic patterns generates $10 \%$ of the total traffic to the hotspot core and the rest 90\% utilizes the skewed 2 and skewed 3 traffic patterns mentioned in table 3-1 respectively. The skewed hotspot3 and skewed hotspot 4 considers a $20 \%$ of traffic to the hotspot coupled with skewed 2 and skewed 3 traffic patterns respectively. This kind of patterns captures the both high frequency communication with some central authority in the CMP like a scheduler or controller via the hotspot pattern as well as skewed core to memory interactions.

For the real application based traffic, parallel GPU applications like MUM, BFS, CP, RAY and LPS [26] are mapped to 20, 4, 4, 4 and 16 cores respectively. These cores are considered to be GPUs occupying 12 clusters. Remaining 4 clusters are considered to have memory cores, which contain the data for the applications mapped to the GPU cores. Then the bandwidth requirement is determined using actual core to memory interaction from profiling these applications in GPGPUSim [27], using GPU-memory bandwidth of 128B flit-size at 700MHz. These particular benchmarks are chosen as BFS and MUM show significant speedup with increase in 
GPU-memory bandwidth, while the other others do not. Hence, this combination represents an actual multi-core chip running multiple parallel applications. The peak bandwidth and packet energy values for these traffic patterns are shown in Figure 3-5. In all the cases the peak bandwidth of the $d$-HetPNoC is better than the Firefly architecture. This is because of the insufficient bandwidth allocation in the Firefly architecture for the high bandwidth communications. However, the degradation in energy and bandwidth is less for the $d$-HetPNoC as it can allocate high bandwidth to communication channels that need it unlike the baseline Firefly. The same trend is observed regardless of the actual percentage traffic with the hotspot.

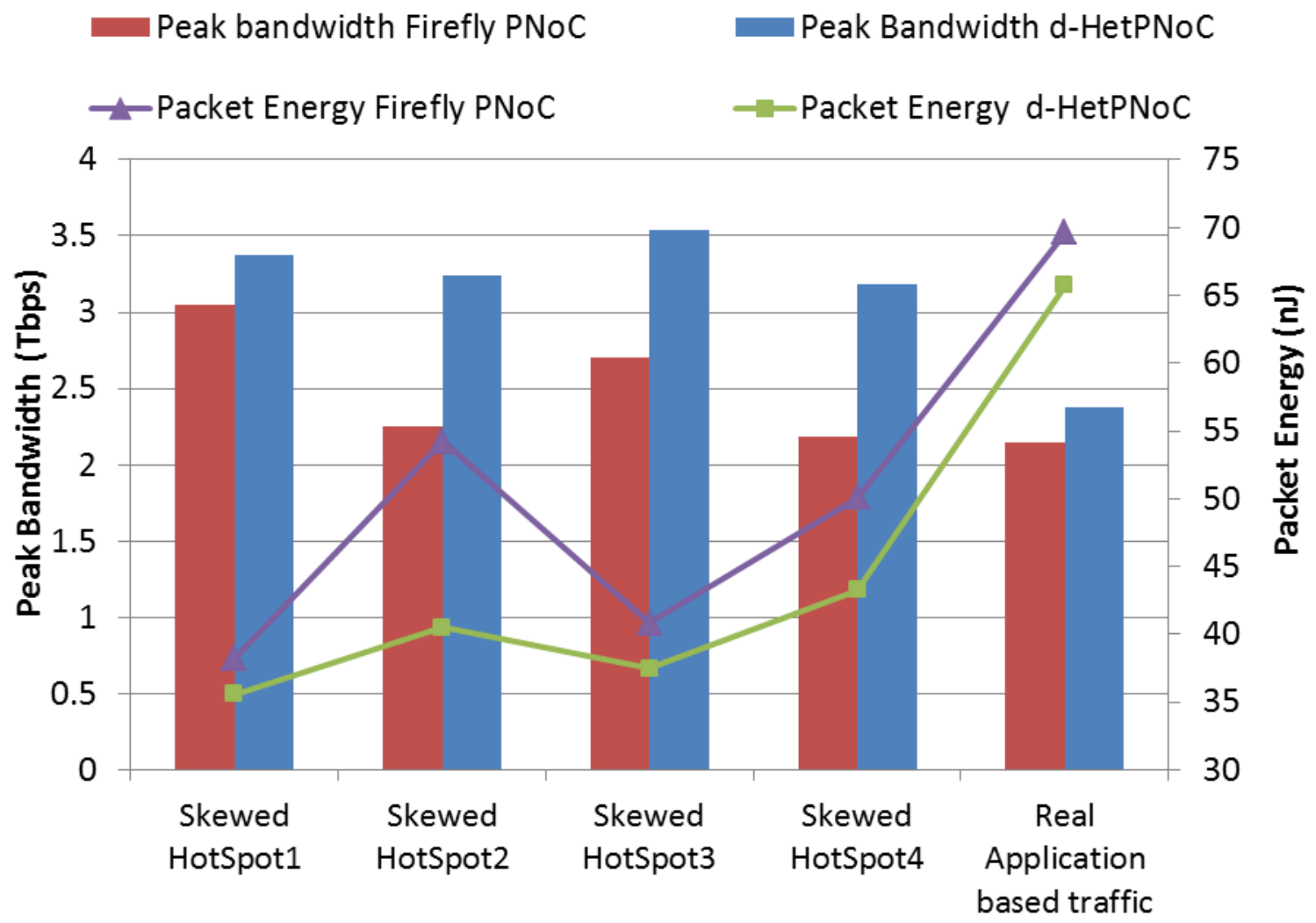

Figure 3-5: Peak Core Bandwidth and Packet Energy for Firefly PNoC for synthetic and real application based traffic scenarios 
In case of the real application based traffic, the interaction between the memory clusters and some of the core clusters require higher bandwidth. This results in a lower peak bandwidth for Firefly compared to the $d$-HetPNoC as it cannot provide the high bandwidth to the clusters that need it.

\subsubsection{Area Overhead}

The dynamic PNoC gives the flexibility to dynamically allocate bandwidth for data communications. However, it incurs an overhead in terms of photonic waveguides and electro-optic devices to enable the dynamic allocation scheme.

Dynamic bandwidth allocation for data communication can be achieved by assigning different number of wavelengths between any pair of photonic routers. Let $\lambda_{N}$ be the total wavelengths required to support all data communications between photonic routers. For dynamic PNoC, number of waveguides, $N_{W D}$ is proportional to the total bandwidth requirement and is given by $\left\lceil\lambda_{N} / \lambda_{W}\right\rceil .\lceil\cdot\rceil$ Function gives the next higher integer value in case the division results in a floating point number. Total number of modulators, $T_{M D}$ for dynamic PNoC is the sum total of the modulators required for data waveguide(s) $N_{M D D}$, reservation waveguide(s) $N_{M R D}$ and control waveguide $N_{M C D}$. It is given by formula below:

$T_{M D}=N_{M D D}+N_{M R D}+N_{M C D}$

Since each photonic router needs to have the capability to modulate on any wavelength in any waveguide, $N_{M D D}$ is given as product of number of photonic 
routers $N_{P R}$, maximum number of wavelengths in a waveguide and number of waveguides.

$$
N_{M D D}=N_{P R} * \lambda_{W} * N_{W D}
$$

In reservation waveguide, each photonic router writes to a dedicated waveguide. Hence $N_{M R D}$ is given as product of number of photonic routers and maximum number of channels per waveguide.

$$
N_{M R D}=N_{P R} * \lambda_{W}
$$

In control waveguide, each photonic router on receiving the token can write to the control waveguide using all the channels in the waveguide. $N_{M C D}$ is given as product of number of photonic routers and maximum number of channels per waveguide in control waveguide.

$$
N_{M C D}=N_{P R} * \lambda_{W}
$$

Putting the values of equation $6,7,8$ in equation 5 we get:

$$
T_{M D}=N_{P R} * \lambda_{W} * N_{W D}+2 * N_{P R} * \lambda_{W}
$$

Each photonic router in Firefly Architecture writes on its dedicated waveguide for data communication. Hence number of waveguides, $N_{W F}$ is equal to number of photonic routers. The number of wavelengths required per waveguide, $\lambda_{N F}$ for achieving the same total bandwidth as dynamic PNoC is given by $\left(\lambda_{N} / N_{W F}\right)$.Total number of modulators, $T_{M F}$ for Firefly Architecture is the sum total of the 
modulators required for data waveguide(s), $N_{M D F}$ and reservation waveguide(s) $N_{M R F}$. It is given by formula below:

$T_{M F}=N_{M D F}+N_{M R F}$

Each photonic router in data waveguide writes to a dedicated waveguide on $\lambda_{N F}$ channels. Hence $N_{M D F}$ is given as product of number of photonic routers $N_{P R}$ and $\lambda_{N F}$.

$N_{M D F}=N_{P R} * \lambda_{N F}$

In reservation waveguide, each photonic router writes on all channels in its dedicated waveguide. Hence $N_{M R F}$ is given as product of number of photonic routers and maximum number of channels per waveguide.

$N_{M R F}=N_{P R} * \lambda_{W}$

Putting the values of equation 11 and 12 in equation 10 we get:

$T_{M F}=N_{P R} * \lambda_{N F}+N_{P R} * \lambda_{W}$

For dynamic PNoC, total number of detectors, $T_{D M D}$ for dynamic PNoC is the sum total of the detectors required for data waveguide(s) $N_{D M D D}$, reservation waveguide(s) $N_{D M R D}$ and control waveguide $N_{D M C D}$. It is given by formula below:

$T_{D M D}=N_{D M D D}+N_{D M R D}+N_{D M C D}$

In data waveguides, since each photonic router needs to have the capability to receive on any wavelength in any waveguide, $N_{D M D D}$ is given as product of number 
of photonic routers $N_{P R}$, maximum number of channels per waveguide in a waveguide and number of waveguides.

$$
N_{D M D D}=N_{P R} * \lambda_{W} * N_{W D}
$$

In reservation waveguide, each photonic router reads from all waveguides except the one to which it writes. Hence $N_{D M R D}$ is given as:

$$
N_{D M R D}=N_{P R} * \lambda_{W} *\left(N_{P R}-1\right)
$$

In control waveguide, each photonic router can receive on all the channels in the waveguide. $N_{D M C D}$ is given as product of number of photonic routers and maximum number of channels/wavelengths in control waveguide.

$$
N_{D M C D}=N_{P R} * 64
$$

Putting the values of equations 15,16 and 17 in equation 14 we get:

$$
T_{D M D}=N_{P R} * \lambda_{W} * N_{W D}+N_{P R} * \lambda_{W} *\left(N_{P R}-1\right)+N_{P R} * \lambda_{W}
$$

Total number of detectors, $T_{D M F}$ for Firefly Architecture is the sum total of the detectors required for data waveguide(s), $N_{D M D F}$ and reservation waveguide(s) $N_{D M R F}$. It is given by formula below:

$T_{D M F}=N_{D M D F}+N_{D M R F}$

Each photonic router in Firefly architecture reads on $\lambda_{N F}$ channels in all data waveguides except its own write waveguide. Hence $N_{D M D F}$ is given as: 


$$
N_{D M D F}=N_{P R} * \lambda_{N F} *\left(N_{P R}-1\right)
$$

In reservation waveguide, each photonic router reads from all waveguides except the one to which it writes. Hence $N_{D M R F}$ is given as:

$$
N_{D M R F}=N_{P R} * \lambda_{W} *\left(N_{P R}-1\right)
$$

Putting the values of equations 20 and 21 in equation 20 we get:

$$
T_{D M F}=N_{P R} * \lambda_{N F} *\left(N_{P R}-1\right)+N_{P R} * \lambda_{W} *\left(N_{P R}-1\right)
$$

We consider MRR's having radius of $5 \mu \mathrm{m}$ [28]. Hence the total area, $A_{D}$ required by electro-optic devices in dynamic $\mathrm{PNoC}$ is given by:

$$
A_{D}=\left(T_{M D}+T_{D M D}\right) * \pi *(5 \mu m)^{2}
$$

Total Area, $A_{F}$ required by electro-optic devices in Firefly architecture is given by:

$$
A_{F}=\left(T_{M F}+T_{D M F}\right) * \pi *(5 \mu m)^{2}
$$

We consider a 64 core-16 cluster system to study the increase in area with increase in total bandwidth requirement. The total modulator/demodulator area for $d$ HetPNoC and Firefly are $1.608 \mathrm{~mm}^{2}$ and $1.367 \mathrm{~mm}^{2}$ respectively for the configuration with 64 data wavelengths studied in this work. Summing equation 9 and equation 18 would give the total number of modulators and demodulators needed for data waveguides. From Figure 3-6 we observe that when the aggregate data bandwidth or total number of wavelengths for data communication is less, the area overhead is minimal. When bandwidth requirement is small, the number of 
data waveguides, $N_{W D}$ is small. Dynamic PNoC provides the feature of dynamically allocating the wavelengths. Hence it needs to provide the flexibility to write to any wavelength within any waveguide. With less number of waveguides, photonic router would need the capability to write to fewer waveguides and hence the hardware overhead is less. As the total bandwidth requirement increases, $N_{W D}$ increases. Since the photonic router may need to write to any WDM channel within any waveguide depending on the dynamic wavelength allocation, the number of modulators needed to support data communication also increases.

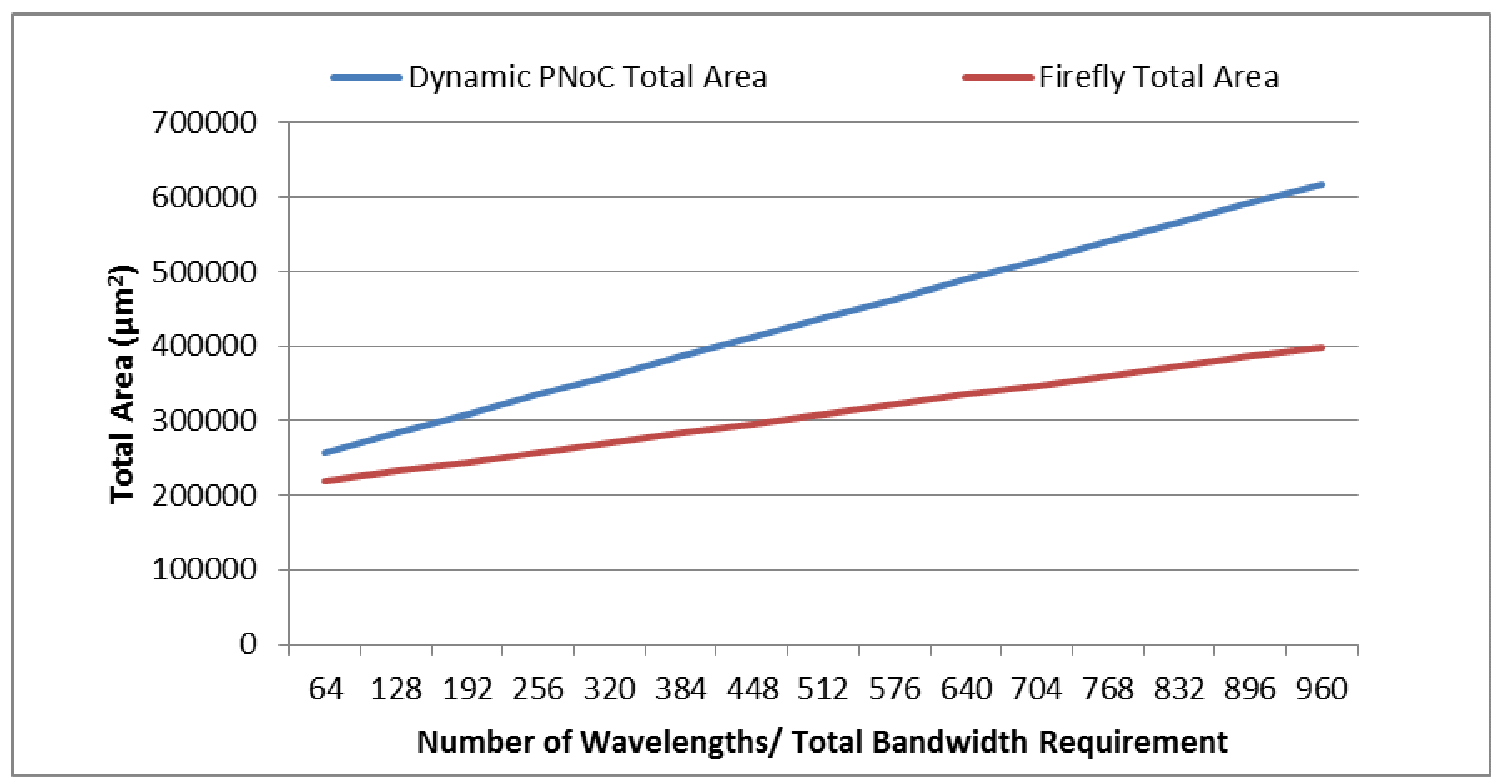

Figure 3-6: Comparison of total area of $d$-HetPNoC and Firefly architecture with increase in total bandwidth requirement

This is the major factor for area overhead in $d$-HetPNoC. It can be seen from equation 6 that there is a linear relationship between the modulators needed for data communication in $d$-HetPNoC and the total bandwidth requirement. The other although less significant, factor for area overhead is the use of dedicated control 
waveguide for circulating the token between photonic routers allowing them to dynamically use the available wavelengths for data communication. This factor remains constant and is independent of the aggregate data bandwidth requirement. While the total aggregate data bandwidth remains the same between the crossbar based Firefly and the $d$-HetPNoC, the total area dedicated to the modulators and demodulators are higher in $d$-HetPNoC due to the flexibility of all clusters being able to write to any wavelength in the data waveguides.

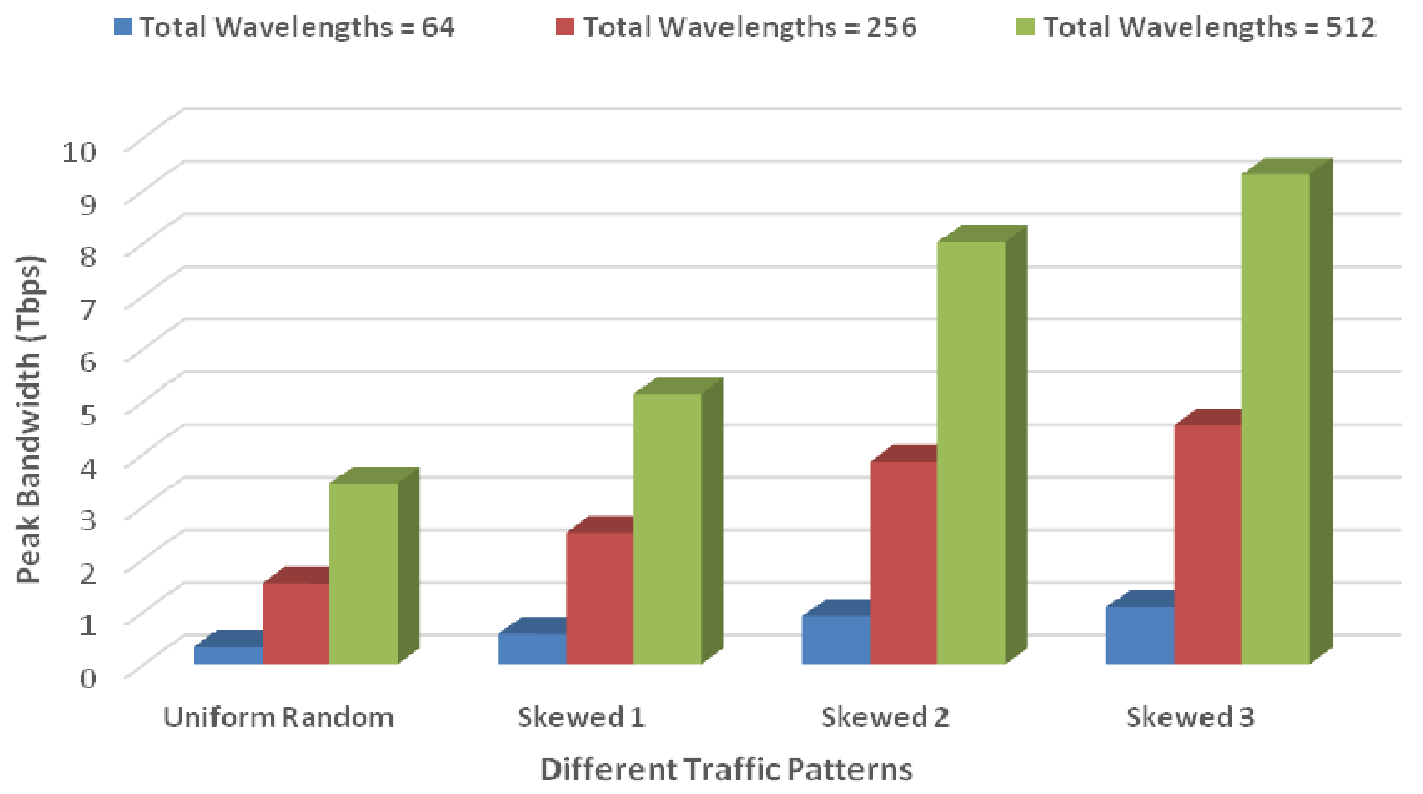

(a) 


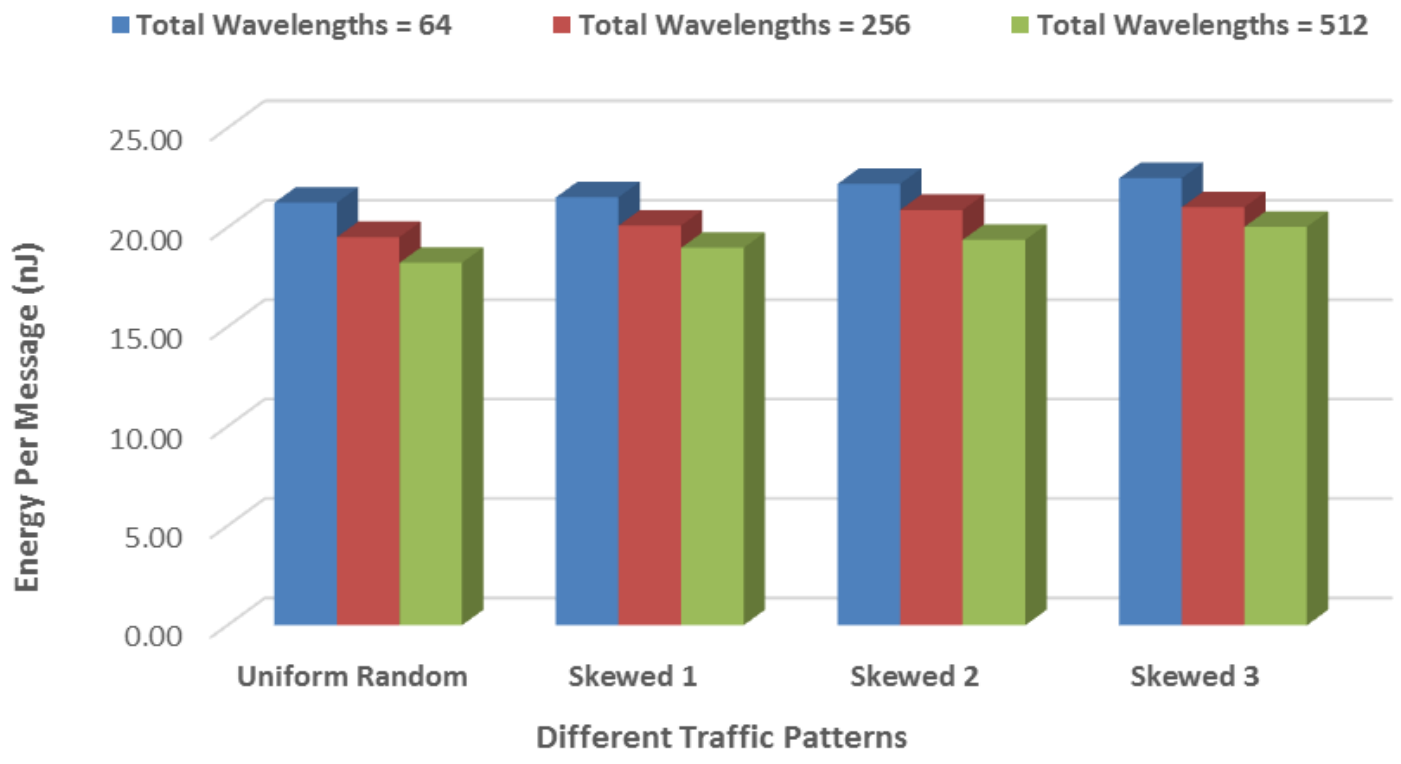

(b)

Figure 3-7: Comparison of (a) Peak Core Bandwidth (b) Energy Per Message of $d$-HetPNoC for BW Set 1 (Total Wavelengths = 64), BW Set 2 (Total Wavelengths $=256$ ) and BW Set 3 (Total Wavelengths $=512$ ) for Unif orm Random and Skewed Traffic Patterns

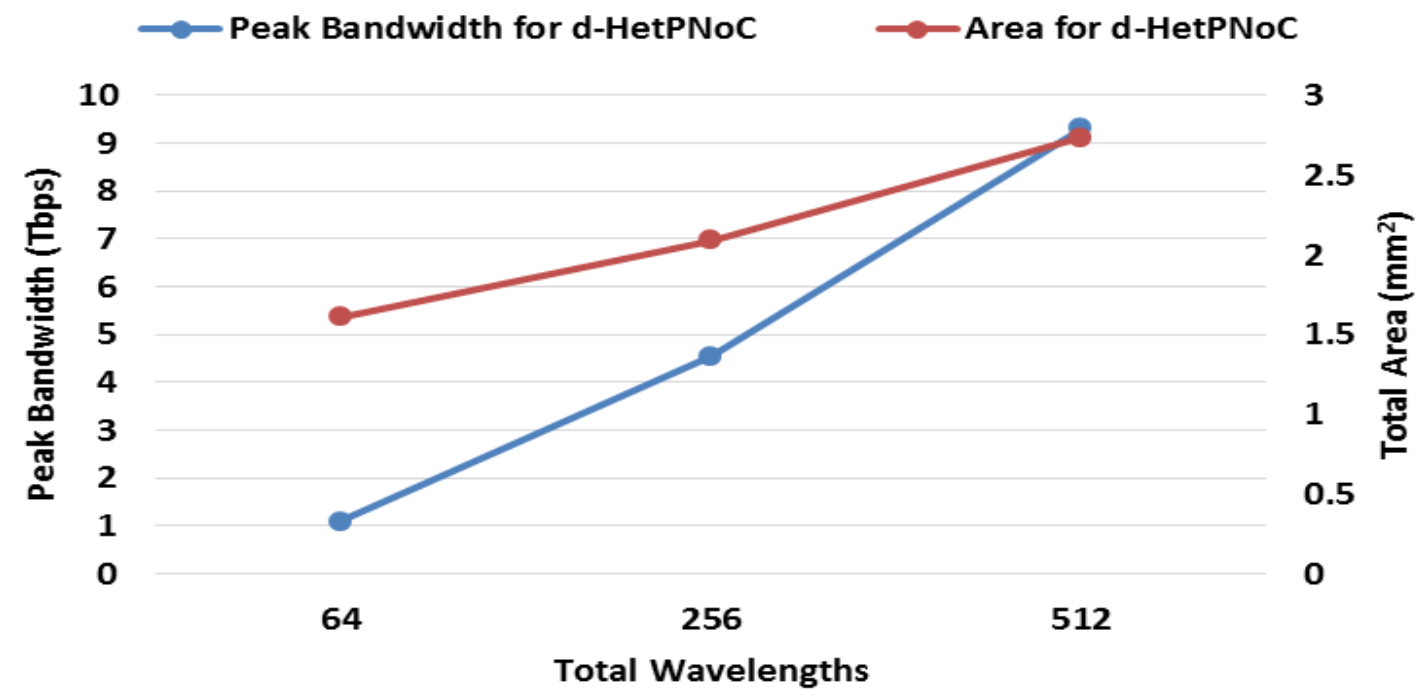

Figure 3-8: Effect of increase in total number of wavelengths on Peak Bandwidth and Area for $d$-HetPNoC for Skewed 3 traffic pattern. 


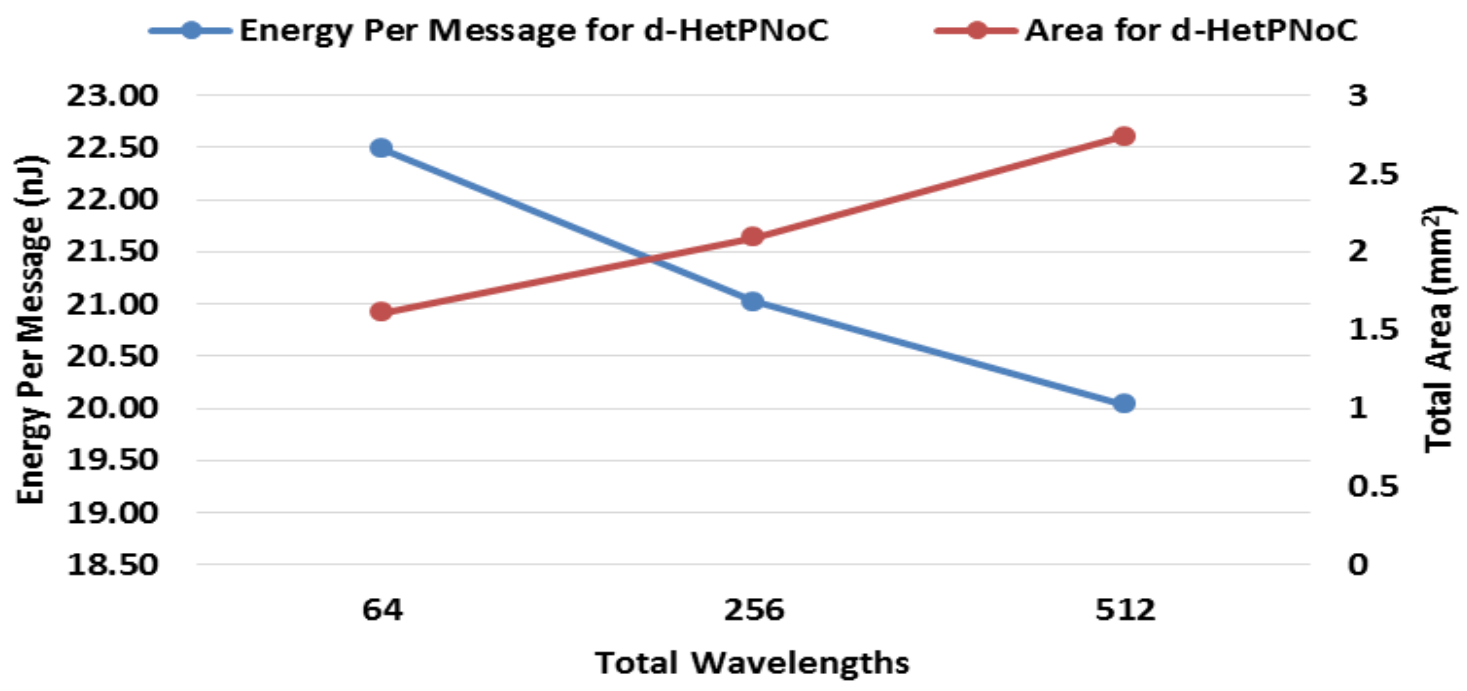

Figure 3-9: Effect of increase in total number of wavelengths on Energy per Message and Area for $d$-HetPNoC for Skewed 3 traffic pattern.

Though area overhead of $d$-HetPNoC as compared to Firefly increases with increase in total bandwidth requirement, $d$-HetPNoC also provides a corresponding performance improvement as shown in figure 3-7. From figure 3-7 we can infer that for all traffic patterns, there is a significant improvement in peak bandwidth and decrease in energy per message with increase in total bandwidth requirement. In figure 3-8 we compare the total area against peak bandwidth and in figure 3-9 we compare the total area against energy per message for skewed 3 traffic pattern, as the total number of wavelengths increase from 64 to 512. From figures 3-8 and 3-9 we can infer that as the total wavelength changes from 64 to 512, the total area increases by $70 \%$ but the corresponding increase in peak bandwidth is $751.31 \%$ while the decrease in packet energy is $10.89 \%$. While the bandwidth increases significantly the packet energy does not change much. Peak bandwidth is given as product of throughput, size of the flit and clock frequency. Increase in total number 
of wavelengths signifies that most of the cores are running high bandwidth applications. Since these applications are high bandwidth, more wavelengths are used to carry data on the photonic links. With increase in number of wavelengths to carry data, the number of bits carried in one clock cycle increases. This is achieved by increasing the flit size which contributes to increase in peak bandwidth. With increased number of wavelengths to carry data, there is also an increase in the throughput as more flits reach the destination in less time. These are the major reasons for significant increase in peak bandwidth. The energy dissipation for a single bit transfer remains practically unchanged across the photonic interconnects resulting in little decrease in overall packet energy.

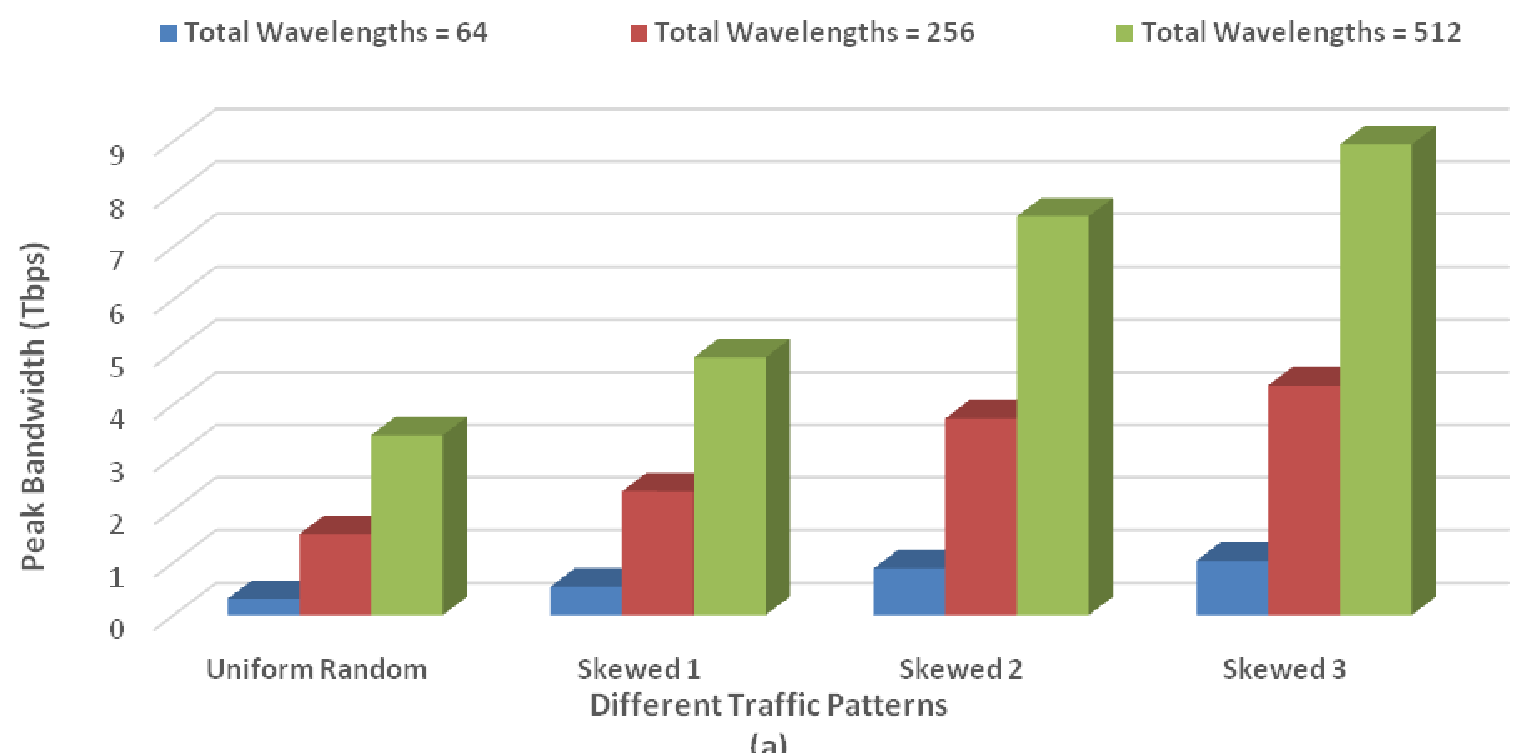

(a) 


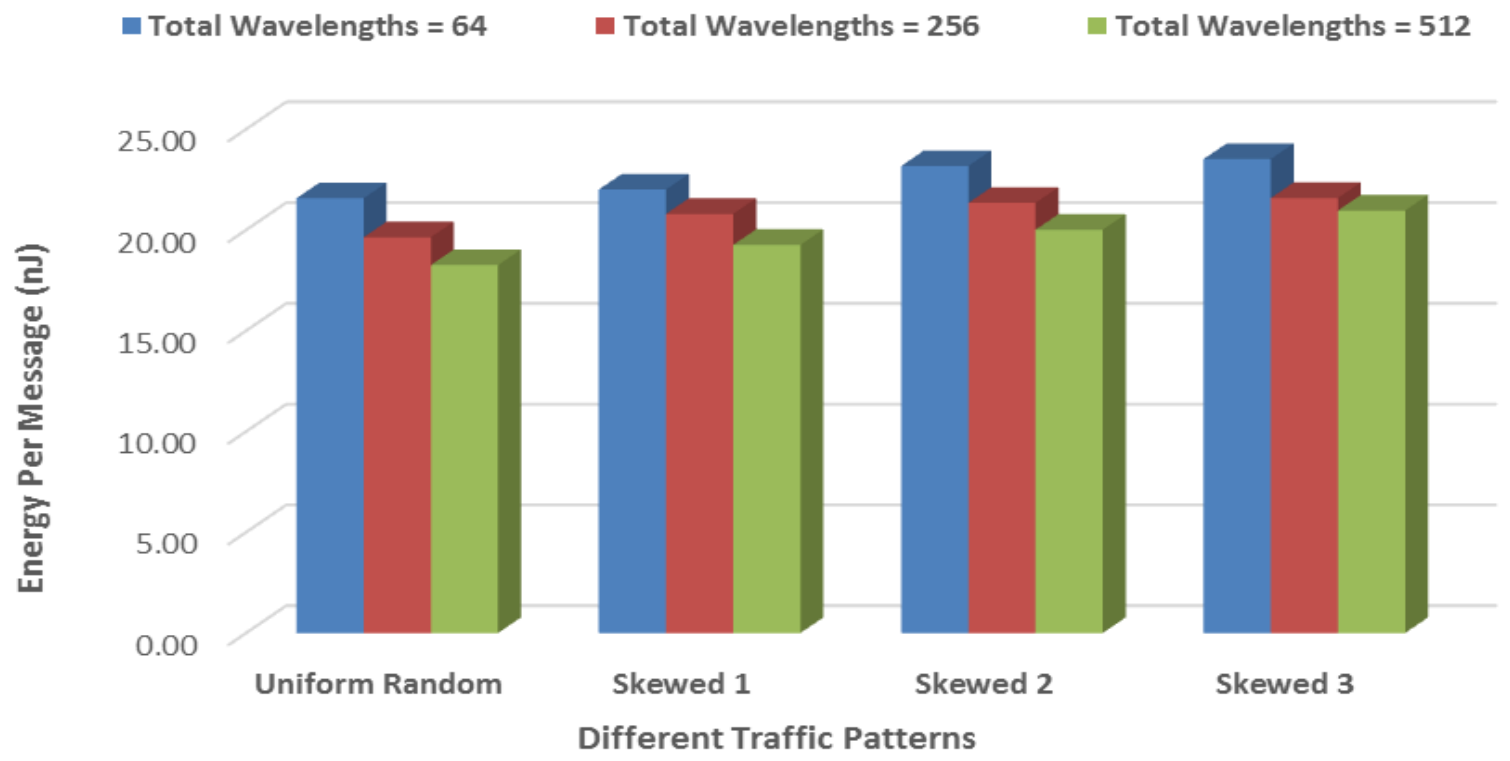

(b)

Figure 3-10: Comparison of (a) Peak Core Bandwidth (b) Energy Per Message of Firefly architecture for BW Set 1 (Total Wavelengths = 64), BW Set 2 (Total Wavelengths $=256)$ and BW Set $3($ Total Wavelengths $=512$ ) for Uniform Random and Skewed Traffic Patterns

Figure 3-10 shows the effect of increase in total bandwidth requirement on peak bandwidth and energy per message for Firefly architecture for different traffic patterns. From figure 3-10 we can infer that as the total wavelength changes from 64 to 256 , the total area increases by $41.17 \%$ but the corresponding increase in total peak bandwidth is $764.52 \%$ and corresponding decrease in energy per message is $10.85 \%$. The reasons for increase in peak bandwidth and decrease in packet energy are same as in case of $d$-HetPNoC. However if we compare figures 3-7 and figure 310 we can see that for all traffic patterns, with increase in total number of wavelengths, the absolute values of peak bandwidth are lower and energy per message are higher than that of $d$-HetPNoC. The reason for higher value of peak bandwidth is $d$-HetPNoC uses the available wavelengths more effectively thus 
causing an increase in throughput and hence causing an increase in peak bandwidth. Energy per message constitutes of photonic link energy and photonic buffer energy. With increased skew in traffic, the routers get congested. With increased skew, since $d$-HetPNoC uses bandwidth more effectively, flits occupy the buffers in routers for a shorter duration as compared to that in Firefly architecture. This causes far less congestion in routers in case of $d$-HetPNoC. Also since flits occupy the buffers for shorter duration, the photonic buffer energy is lesser in case of $d$-HetPNoC thus causing energy per message to be lower in case of $d$-HetPNoC. 


\section{Chapter 4 Conclusion}

In this thesis, we propose a cross-bar based heterogeneous photonic NoC with dynamic bandwidth allocation, which can allocate different bandwidths between different clusters of cores. This dynamic bandwidth requirement is dependent on the type of core and also the application running on the cores.

Section 3.4.1.1 shows that $d$-HetPNoC provides higher peak data bandwidth for all kinds of traffic patterns as compared to Firefly Architecture. The $d$-HetPNoC architecture provides about 8\% increase in peak data bandwidth over Firefly Architecture. Peak data bandwidth also increases with increase in the total bandwidth requirement. Section 3.4.1.2 shows that $d$-HetPNoC dissipates less energy as compared to Firefly architecture thus making the architecture energy efficient. The $d$-HetPNoC dissipates up to 5\% less energy as compared to Firefly architecture. Thus this scheme is demonstrated to achieve higher performance and energy-efficiency for the same overall data bandwidth compared to a homogeneous photonic NoC.

Since $d$-HetPNoC provides DBA, there are certain over heads associated with the scheme. The main over head of $d$-HetPNoC is the area overhead. We envision that this over ahead could be mitigated by restricting the cluster to use wavelengths from certain waveguides. An example would be to restrict a certain photonic router say $\mathrm{PR}_{\mathrm{x}}$ to wavelengths of Waveguide $(\mathrm{x})$ and Waveguide $(\mathrm{x}+1)$. Hence $\mathrm{PR}_{\mathrm{x}}$, would at any point of time would need modulators and de-modulators for all wavelengths in Waveguide $_{(x)}$ and Waveguide $(x+1)$, thus reducing the number of modulators and de- 
modulators. Despite the overheads, architectures with DBA are suitable for future CMPs, which integrates heterogeneous cores like custom logic, GPGPUs, programmable fabrics and memory.

Future work would be to find better ways to effectively manage bandwidth allocation with minimal overheads. 


\section{Reference}

[1] Moore's law -

http://www.intel.com/content/www/us/en/silicon-innovations/moores-lawtechnology.html

[2] E.S. Chung, P.A. Milder, J.C. Hoe and K. Mai, "Single-Chip Heterogeneous

Computing: Does the Future Include Custom Logic, FPGAs, and GPGPUs?," 43rd Annual IEEE/ACM International Symposium on Microarchitecture (MICRO), Dec. 2010, pp.225-236.

[3] Chung, E.S.; Milder, P.A; Hoe, J.C.; Ken Mai, "Single-Chip Heterogeneous Computing: Does the Future Include Custom Logic, FPGAs, and GPGPUs?," Microarchitecture (MICRO), 2010 43rd Annual IEEE/ACM International Symposium on, vol., no., pp.225,236, 4-8 Dec. 2010

doi: 10.1109/MICR0.2010.36

[4] Intel Quick path interconnecthttp://www.intel.com/content/www/us/en/io/quickpath-technology/quickpathtechnology-general.html

[5] Hyper-transport-http://www.hypertransport.org/default.cfm?page=Technology

[6] Pande, P.P.; Grecu, C.; Jones, M.; Ivanov, A; Saleh, R., "Performance evaluation and design trade-offs for network-on-chip interconnect architectures," Computers, IEEE Transactions on , vol.54, no.8, pp.1025,1040, Aug. 2005

doi: 10.1109/TC.2005.134

[7] Yogita A. Sadawarte, Mahendra A. Gaikwad, and Rajendra M. Patrikar. 2011. Comparative study of switching techniques for network-on-chip architecture. In 
Proceedings of the 2011 International Conference on Communication, Computing \& Security (ICCCS '11). ACM, New York, NY, USA, 243-246.

DOI:10.1145/1947940.1947992 http://doi.acm.org/10.1145/1947940.1947992

[8] Carloni, L.P.; Pande, P.; Yuan Xie, "Networks-on-chip in emerging interconnect paradigms: Advantages and challenges," Networks-on-Chip, 2009. NoCS 2009. 3rd ACM/IEEE International Symposium on , vol., no., pp.93,102, 10-13 May 2009 doi: 10.1109/NOCS.2009.5071456

[9] Pavlidis, V.F.; Friedman, E.G., "3-D Topologies for Networks-on-Chip," Very Large Scale Integration (VLSI) Systems, IEEE Transactions on, vol.15, no.10, pp.1081,1090, Oct. 2007 doi: 10.1109/TVLSI.2007.893649

[10] Deb, S.; Ganguly, A; Chang, K.; Pande, P.; Beizer, B.; Heo, D., "Enhancing performance of network-on-chip architectures with millimeter-wave wireless interconnects," Application-specific Systems Architectures and Processors (ASAP), 2010 21st IEEE International Conference on , vol., no., pp.73,80, 7-9 July 2010 doi: 10.1109/ASAP.2010.5540799

[11] Ganguly, A; Chang, K.; Deb, S.; Pande, P.P.; Belzer, B.; Teuscher, C., "Scalable Hybrid Wireless Network-on-Chip Architectures for Multicore Systems," Computers, IEEE Transactions on , vol.60, no.10, pp.1485,1502, Oct. 2011 doi: 10.1109/TC.2010.176

[12] Q. Xu, D. Fattal, and R. Beausoleil, "Silicon microring resonators with 1.5- $\mu \mathrm{m}$ radius," Opt. Express 16, 4309-4315 (2008).

[13] Biberman, A.,"Adiabatic microring modulators" Proc. Optical Fiber 
Communication Conference and Exposition and the National Fiber Optic Engineers conference (OFC/NFOEC), $2013 . \quad$ pp.1-3

[14] D. Ahn, C. Hong, J. Liu, W. Giziewicz, M. Beals, L. Kimerling, J. Michel, J. Chen, and F. Kärtner, "High performance, waveguide integrated Ge photodetectors," Opt. Express 15, 3916-3921 (2007).

[15] A. Shacham et al., "Photonic Network-on-Chip for Future Generations of Chip Multi-Processors", IEEE Transactions on Computers, Vol. 57, no. 9, 2008, pp. 1246-1260.

[16] Heck, M.J.R.; Bowers, J.E., "Energy Efficient and Energy Proportional Optical Interconnects for Multi-Core Processors: Driving the Need for On-Chip Sources," Selected Topics in Quantum Electronics, IEEE Journal of, vol.20, no.4, pp.1,12, JulyAug. 2014 doi: 10.1109/JSTQE.2013.2293271

[17] Bogaerts, W.; Baets, R.; Dumon, P.; Wiaux, V.; Beckx, S.; Taillaert, D.; Luyssaert, B.; Van Campenhout, J.; Bienstman, P.; Van Thourhout, D., "Nanophotonic waveguides in silicon-on-insulator fabricated with CMOS technology," Lightwave Technology, Journal of , vol.23, no.1, pp.401,412, Jan. 2005 doi: 10.1109/JLT.2004.834471(410) 23

[18] K. Ohira, K. Kobayashi, N. Iizuka, H. Yoshida, M. Ezaki, H. Uemura, A. Kojima, K. Nakamura, H. Furuyama, and H. Shibata, "On-chip optical interconnection by using integrated III-V laser diode and photodetector with silicon waveguide," Opt. Express $18,15440-15447$ (2010).

[19] S. Assefa et al., "CMOS-integrated $40 \mathrm{GHz}$ germanium waveuide photodetector for 
on-chip optical interconnects.", Proc. Optcal Fiber Communication - incudes post deadline papers, 2009. OFC 2009. pp. 1-3.

[20] Yan Pan, Prabhat Kumar, John Kim, Gokhan Memik, Yu Zhang, and Alok Choudhary. 2009. Firefly: illuminating future network-on-chip with nanophotonics. In Proceedings of the 36th annual international symposium on Computer architecture (ISCA '09). ACM, New York, NY, USA, 429-440. DOI=10.1145/1555754.1555808 http://doi.acm.org/10.1145/1555754.1555808

[21] A. Joshi et al., "Silicon-Photonic Clos Network for Global On-Chip Communication", Proc. 3rd International Symposium on Networks-on-Chip (NOCS), May 2009, pp. 124-133.

[22] D. Vantrease et al., "Corona: System Implications of Emerging Nanophotonic Technology," Proc. IEEE International Symposium on Computer Architecture (ISCA), 21-25 June, 2008, pp. 153-164.

[23] Y. Xie et al. "Crosstalk Noise and Bit Error Rate Analysis for Optical Network-onChip", Proceedings of IEEE/ACM Design Automation Conference (DAC), 2010, pp. 657-660.

[24] P. Pande,C. Grecu, M. Jones, A. Ivanov, R. Saleh, "Performance evaluation and design trade-offs for network-on-chip interconnect architectures," , IEEE Transactions on Computers, vol.54, no.8, pp.1025-1040, Aug. 2005

[25] S. Che et. al., "Rodinia: A Benchmark Suite for Heterogeneous Computing", In Proceedings of the 2009 IEEE International Symposium on Workload Characterization (IISWC), Washington, DC, USA, 44-54

[26] CUDA Toolkit Documentation, URL: http://docs.nvidia.com/cuda/cuda- 
samples/

[27] A. Bakhoda, G. L. Yuan, W. W. L. Fung, H. Wong, and T. M. Aamodt, "Analyzing CUDA Workloads Using a Detailed GPU Simulator", (ISPASS), 2009, pp. 163-174.

[28] P. Dong et. al., "Tunable high speed silicon microring modulator,", Conference on Lasers and Electro-Optics (CLEO) and Quantum Electronics and Laser Science Conference (QELS), May 2010, pp.1,2.

[29] Circuits MultiProjets, URL: $\underline{\text { http://cmp.imag.fr }}$

[30] K. Preston et al., "Performance Guidelines for WDM Interconnects Based on Silicon Microring Resonators," Proc. Laser and Electro-Optics(CLEO), 2011, pp. 1-2.

[31] A. Mishra, N. Vijaykrishnan and C. R. Das, "A case for Heterogeneous On-Chip Interconnects for CMPs", Proc. of International Symposium Computer Arhitecture (ISCA), 2011. pp. 389-400.

[32] A. K. Mishra, O. Mutlu, and C. R. Das. 2013. A heterogeneous multiple networkon-chip design: an application-aware approach. In Proceedings of the 50th Annual Design Automation Conference (DAC '13). ACM, New York, NY, USA, , Article 36 , 10 pages. 